Check for updates

Cite this: Mater. Chem. Front., 2020, 4, 354

Received 12th August 2019, Accepted 9th November 2019

DOI: 10.1039/c9qm00517j

rsc.li/frontiers-materials

\title{
Application of organic-graphene hybrids in high performance photodetectors
}

\author{
Jie Liu, Qiu Liang, Ruoyu Zhao, Shengbin Lei (D) * and Wenping Hu (D)*
}

\begin{abstract}
High performance photodetectors play important roles in the improvement of innovative technologies in many fields, for instance, medicine, military, optical communication, environment monitoring, etc. Graphene functionalized with photo-active units has been investigated intensively due to the unique optical and electronic properties of the graphene material, which remarkably sustains and expands the graphene boom. This review focuses on the research progress in applications of organic-graphene hybrid films in photodetectors. We discuss the sensitization mechanisms of photo-response, fabrication of hybrid material devices and sensitization of graphene by different organic molecules. The organicgraphene hybrid materials hold great promise to realize high performance photodetectors with high responsivity and a fast response.
\end{abstract}

\section{Introduction}

Graphene, which is the most famous single-atom-thick twodimensional (2D) material, has a honeycomb lattice composed of pure $\mathrm{sp}^{2}$-bonded carbon atoms. ${ }^{1,2}$ It has attracted intense attention in recent years due to its unique excellent properties such as strong light absorption as a single atomic layer, ${ }^{3,4}$ high carrier mobility, ${ }^{1,5}$ good transparency from the visible to the

Tianjin Key Laboratory of Molecular Optoelectronic Science, Department of Chemistry, School of Science \& Collaborative Innovation Center of Chemical Science and Engineering (Tianjin), Tianjin University, Tianjin, 300072, P. R. China. E-mail: shengbin.lei@tju.edu.cn,huwp@tju.edu.cn infrared range, ${ }^{6}$ tuneable optical properties, ${ }^{7-14}$ high thermal conductivity, good mechanical flexibility and large specific surface area. ${ }^{15-18}$ These outstanding optical, electronic and mechanical properties of graphene made it a great promising material in photodetectors. However, the intrinsically low absorption cross-section and zero band gap of graphene pose a serious problem for realization of high-performance photodetectors.

To improve the performance of graphene-based photodetectors, a facile sensitization method is urgently needed. Recently, more and more researchers have focused on developing graphene-based hybrid nanostructures to overcome the shortcomings of graphene, which provides new opportunities to fabricate high-performance optoelectronic devices. Hybrid nanostructures, which combine the

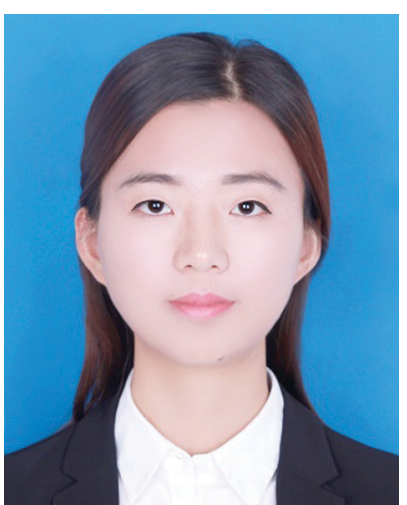

Jie Liu
Jie Liu grew up in Henan province. In 2019, she received her PhD from the Department of Chemistry, Tianjin University under the supervision of Prof. Shengbin Lei. She is currently pursuing her postdoctoral research at the Key Laboratory of Organic Solids, Institute of Chemistry Chinese Academy of Sciences (ICCAS). Her research interests are in the design and synthesis of $2 D$ functional materials and characterization of their optoelectronic properties.

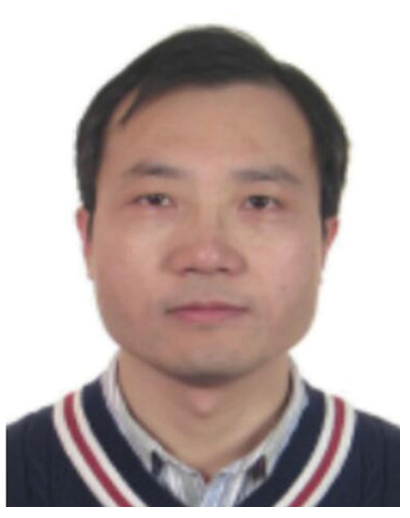

Shengbin Lei
Shengbin Lei received his PhD from ICCAS in 2002. Then he was an assistant researcher in 2002 and an associate researcher in 2005 at the ICCAS. After his postdoctoral research at $K U$ Leuven - University of Leuven, Belgium, he joined Harbin Institute of Technology in 2009 as a professor. Since February 2015, he has been a professor at Tianjin University. His research interest includes surface confined dynamic covalent chemistry and twodimensional organic materials and related device applications. He has published more than 130 peer reviewed papers with over 4000 citations. 
unique properties of individual constituents, usually consist of two or more components with distinct functionality exhibiting optimized optoelectronic performance..$^{7,14,19-24}$ Transition metal disulfides (TMDs, such as $\mathrm{MoS}_{2}, \mathrm{WS}_{2}$, etc.), ${ }^{25-27}$ quantum dots (such as $\mathrm{PbS}$ ), ${ }^{28}$ gold nanoparticles ${ }^{29,30}$ and perovskites ${ }^{31,32}$ have been applied in sensitization of graphene and the responsivity of the device is significantly improved. In comparison with the above mentioned inorganic materials, organic-graphene hybrid films can be fabricated easily through a simple solution process over large areas on flexible substrates, and the abundance of organic materials also enables diverse choice to fabricate organic-graphene hybrids, which have attracted intense interest from researchers.

Graphene-organic hybrid materials are composites of graphene and organic photoactive materials, such as organic dyes, organic small molecules or polymer semiconductors. ${ }^{33-46}$ Light absorption in the spectral region of such graphene-organic hybrids is tuneable by appropriately selecting the property of photoactive materials, which makes them more fascinating in the optoelectronic field. This review aims to give an overview of the progress in graphene-organic hybrid materials and their applications in high performance photodetectors. We will discuss from the aspects of photosensitization mechanisms, methods for hybrid material preparation, and the fabrication and characterization of devices. Given the large library of organic molecules, we believe that organic-graphene hybrid materials can serve as a versatile platform for high-performance, broadband photodetectors.

\section{Sensitization mechanism of graphene-based photodetectors}

Graphene, which is made out of $\mathrm{sp}^{2}$-hybridized carbon atoms arranged on a honeycomb lattice, is a zero-band-gap semiconductor because the valence and conduction bands touch at the Brillouin zone corners (so-called Dirac or neutrality point). ${ }^{47-49}$

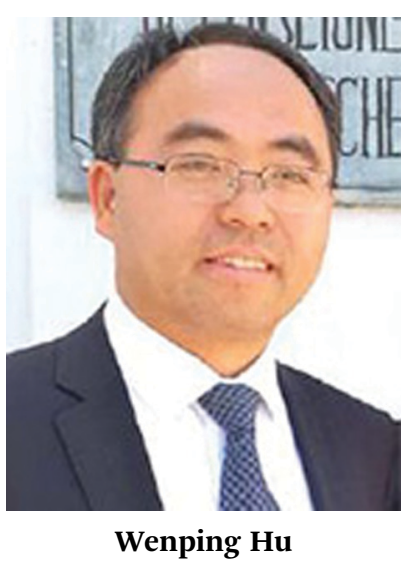

Wenping $\mathrm{Hu}$ is a Professor at Tianjin University and a Cheung Kong Professor of the Ministry of Education, China. He received his PhD from ICCAS in 1999 under the supervision of Prof. Daoben Zhu and Prof. Yunqi Liu. Then he joined Osaka University and Stuttgart University as a research fellow of the Japan Society for the Promotion of Sciences and an Alexander von Humboldt fellow, respectively. In 2003 he joined ICCAS as a full professor. He has worked for Tianjin University since 2013 and was promoted to the Vice President of the university in 2016. He focuses on organic optoelectronics, and has published more than 500 peer reviewed papers with $\sim 18000$ citations ( $\mathrm{H}$ index $=68$ ).

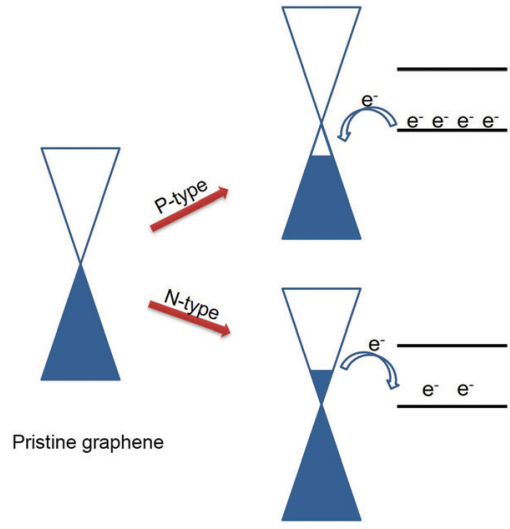

Fig. 1 Energy band diagram after establishing contact between graphene and the photo-active molecule.

As a result, the zero band gap nature of graphene also leads to a low lifetime of carriers, ${ }^{50,51}$ thus photodetectors made of pure graphene lack gain mechanisms (e.g., carrier multiplication), which limits the improvement of some key parameters of photodetectors, for instance, the responsivity, noise equivalent power and quantum efficiency. As graphene is an intrinsic, atomically thin two-dimensional material, its conductance is very sensitive to electrostatic perturbation at the interface, thus making graphene a particularly promising material for high-gain photo-detection through a photogating effect. ${ }^{52}$ When graphene is functionalized by organic molecules, with a high absorption cross-section and spectrally tuneable absorption, the photogenerated charges in the organic molecules can transfer to graphene, while oppositely charged carriers remain trapped in the organic layer. ${ }^{53,54}$ The n-doping or p-doping effect are produced on graphene due to the properties of modified layer (Fig. 1). The carriers, which are trapped in the organic layer, lead to a photogating effect, where the presence of these charges changes the resistance of graphene through capacitive coupling. The high carrier mobility of graphene and the recirculation of charge carriers during the lifetime leads to ultrahigh gain, which improves the quantum efficiency and photocurrent. ${ }^{5,56}$ The charge transfer is enhanced because of the difference in Fermi level, which increases the number of mobile charge carriers present in the graphene. Charge carriers are generated from the incident photon and electrons move from the graphene layer to the sensitization layer when the Fermi level of graphene is higher, whereas the holes transferred to the opposite direction. Excess carriers (electrons or holes) are injected into graphene and drift to the drain electrode under the bias between the source and drain, and the drift time is $\tau_{\text {transit}}$. Because of the accumulation of carriers (electrons or holes) in graphene, the recombination process in the sensitized layer is hindered. Therefore, benefited from the ultrahigh mobility value of graphene $\left(1000 \mathrm{~cm}^{2} \mathrm{~V}^{-1} \mathrm{~s}^{-1}\right)$ the recombination time ( $\left.\tau_{\text {lifetime }}\right)$ in the activated layer is much longer than the transit time of the carriers (on the order of milliseconds compared to on the order of picoseconds). Then, the gain of photodetectors is given by $G=\eta \tau_{\text {lifetime }} / \tau_{\text {transit }}$, in which $\eta$ is the quantum efficiency. $^{57}$ 


\section{The fabrication of device}

With the development of synthetic techniques and device fabrication methods, the rational integration of nanomaterials into various hybrid nanostructures with enhanced photodetection performances has been achieved, as summarized in Table 1.

\subsection{The choice of substrate}

The type of substrate has a great effect on the performance of the device. Typically, a $\mathrm{Si} / \mathrm{SiO}_{2}$ substrate is used when the photodetectors are fabricated, and a heavily doped Si wafer is employed as the gate electrode and a thermally grown $300 \mathrm{~nm}$ $\mathrm{SiO}_{2}$ layer above it acts as a gate dielectric. ${ }^{58-61}$ The carrier density, and thus the conductivity of the channel can be modulated by an electric field applied on the gate. The surface of the $\mathrm{SiO}_{2}$ dielectric can be modified with self-assembling monolayers composed of, e.g., n-octadecyltrimethoxysilane (ODTS) to decrease charge trapping caused by defects.

Conventional photodetectors based on inorganic materials are usually built on rigid and planar substrates, which limits their application in flexible devices. Phototransistors based on organic-graphene hybrid materials are easy to integrate with low-temperature processable flexible substrates over large areas, for instance, polyethylene terephthalate (PET) and polyimide (PI). ${ }^{62-66}$ Because of its good mechanical flexibility, graphene finds an irreplaceable position in the fabrication of flexible devices, and hence it is clear that a substrate with flexibility plays a very important role in developing a new type of device. Ki-Seok An et al. have successfully fabricated various graphene-based photodetectors via simple and efficient vapor-phase metalation of porphyrin on PET films and facilitated the development of new architectures for flexible graphene-organic devices. ${ }^{43}$

\subsection{Preparation of graphene}

Both pristine graphene and chemically functionalized graphene are highly attractive for applications in optoelectronics and photoenergy conversion, due to their excellent electronic and optical properties as well as tuneable work functions. ${ }^{67-70}$ Synthesis of graphene is essential for its practical application in electronics and optoelectronics. Graphene can be obtained in different forms, and the most commonly used types are the exfoliated graphene layers $^{71-75}$ and graphene grown by the chemical vapor deposition (CVD) method on various substrates. ${ }^{76-80}$

The quality of graphene has a great influence on the electronic properties of hybrid materials. Mechanically exfoliated graphene is relatively clean in comparison with CVD-grown graphene and is widely used in the experimental studies of partial theoretical calculations. $^{81-84}$ In addition to that, electrochemical exfoliated graphene can be easily fabricated into a large sized paper like form by applying brush painting of a concentrated graphene ink, which shows great promise for the industrial-scale synthesis of highquality graphene for numerous advanced applications. ${ }^{85}$

In particular, a large area, high-quality graphene film grown by the chemical vapor deposition (CVD) method is more promising for extensive applications because of its flexibility, high transparency, and electrical conductivity, and most importantly due to the fact that the number of layers can be controlled by adjusting growth conditions. ${ }^{86,87}$ After the CVD graphene is separated from the metallic catalyst where it is grown, it can be easily transferred to a dielectric substrate, which is a

Table 1 The key parameters of several recently reported organic-graphene hybrid photodetectors

\begin{tabular}{|c|c|c|c|c|c|c|c|c|c|c|}
\hline Photoactive $\mathrm{m}$ & laterials & Graphene & $\begin{array}{l}\text { Fabrication of } \\
\text { hybrid material }\end{array}$ & Substrate & $\begin{array}{l}\text { Doping } \\
\text { type }\end{array}$ & $\begin{array}{l}\text { Responsivity } \\
\left(\mathrm{A} \mathrm{W}^{-1}\right)\end{array}$ & $\begin{array}{l}\text { Detectivity } \\
\text { (Jones) }\end{array}$ & $\begin{array}{l}\text { Maximum } \\
\text { absorption } \\
\text { wavelength } \\
(\mathrm{nm})\end{array}$ & $\begin{array}{l}\text { Determined } \\
\text { wavelength } \\
(\mathrm{nm})\end{array}$ & Ref \\
\hline \multirow[t]{6}{*}{$\begin{array}{l}\text { Rhodamine } \\
\text { derivatives }\end{array}$} & R6G & $\begin{array}{l}\text { Exfoliated } \\
\text { graphene }\end{array}$ & $\begin{array}{l}\text { Dip-pen nano- } \\
\text { lithography (DPN) }\end{array}$ & $\mathrm{Si} / \mathrm{SiO}_{2}$ & $\mathrm{n}$ & - & - & - & - & 33 \\
\hline & R6G & CVD & Drop-casting & \multirow{5}{*}{$\begin{array}{l}\mathrm{Si} / \mathrm{SiO}_{2} \\
\text { (ODTS) }\end{array}$} & $\mathrm{p}$ & $\sim 460$ & \multirow[t]{4}{*}{$10^{16}$} & 530 & \multirow[t]{4}{*}{$400-980$} & 34 \\
\hline & Rh110 & CVD & Dip-coating & & $\mathrm{p}$ & 283 & & 490 & & 35 \\
\hline & Rh101 & CVD & Dip-coating & & $\mathrm{n}$ & 455 & & 565 & & \\
\hline & Rh800 & CVD & Dip-coating & & $\mathrm{p}$ & 1031 & & 685 & & \\
\hline & $\begin{array}{l}\text { Rh110/Rh101/ } \\
\text { Rh800 }\end{array}$ & CVD & Drop-casting & & & $10^{3}$ & $10^{10}$ & - & $<760$ & \\
\hline \multirow[t]{3}{*}{ Self-assembly } & D-A type & CVD & - & - & - & - & - & 740 & 710 & \multirow[t]{2}{*}{44} \\
\hline & molecule & CVD & Spin-casting & $\begin{array}{l}\text { ITO/ } \\
\text { PEDOT:PSS }\end{array}$ & - & - & - & - & - & \\
\hline & $\begin{array}{l}\mathrm{ml}^{-\mathrm{C}_{8}} \text {-BTBT } \\
\text { fl-C }_{8}-\text { BTBT }\end{array}$ & $\begin{array}{l}\text { CVD } \\
\text { CVD }\end{array}$ & $\begin{array}{l}\text { CVD } \\
\text { CVD }\end{array}$ & $\mathrm{Si} / \mathrm{SiO}_{2}$ & $\mathrm{p}$ & $\begin{array}{l}1.57 \times 10^{4} \\
4.76 \times 10^{5}\end{array}$ & - & 360 & 355 & 42 \\
\hline \multirow{3}{*}{$\begin{array}{l}\text { Tetraphenyl- } \\
\text { porphyrin } \\
\text { (H2TPP) }\end{array}$} & $\mathrm{H}_{2} \mathrm{TPP}$ & CVD & Thermal & \multirow{3}{*}{$\begin{array}{l}\mathrm{Si} / \mathrm{SiO}_{2} \\
(\mathrm{PET})\end{array}$} & $\mathrm{n}$ & 0.22 & - & - & - & \multirow[t]{3}{*}{43} \\
\hline & ZnTPP & CVD & evaporation (TE) & & $\mathrm{p}$ & 0.54 & - & - & - & \\
\hline & AlTPP & CVD & & & & 5.36 & - & - & - & \\
\hline $\begin{array}{l}\text { Ruthenium } \\
\text { polypyridyl }\end{array}$ & Ru-Complex & CVD & Dip-coating & $\mathrm{Si} / \mathrm{SiO}_{2}$ & $\mathrm{n}$ & $10^{5}$ & - & - & - & 37 \\
\hline P3HT & $\begin{array}{l}\text { P3HT/graphene/ } \\
\text { TiO }_{x}\end{array}$ & CVD & Spin-coating & $\mathrm{Si} / \mathrm{SiO}_{2}$ & $\mathrm{p}$ & - & - & - & - & 36 \\
\hline \multirow{3}{*}{$\begin{array}{l}\text { Spiropyran/ } \\
\text { merocyanine } \\
\text { SWCNTs }\end{array}$} & Spiropyran & CVD & Dip-coating & $\mathrm{Si} / \mathrm{SiO}_{2}$ & $\mathrm{n}$ & - & - & 560 & - & 38 \\
\hline & & CVD & Spin-coating & $\mathrm{Si} / \mathrm{SiO}_{2}$ & $\mathrm{n}$ & - & - & - & - & 39 \\
\hline & $\begin{array}{l}\text { Single-walled } \\
\text { carbon nanotubes }\end{array}$ & $\begin{array}{l}\text { Separated } \\
\text { graphene }\end{array}$ & Dip-coating & $\mathrm{Si} / \mathrm{SiO}_{2}$ & - & - & - & - & - & 40 \\
\hline Biomaterials & Cytochrome $c$ & CVD & Print & $\mathrm{Si} / \mathrm{SiO}_{2}$ & $\mathrm{n}$ & $\begin{array}{l}1.39 \times 10^{4} / \\
7.57 \times 10^{4}\end{array}$ & - & - & 340,410 & 41 \\
\hline
\end{tabular}


commonly used method in device fabrication. Then using conventional photolithography and subsequent reactive ion etching (RIE) with $\mathrm{O}_{2}$ plasma etching steps, channels with a certain width can be patterned on the substrate. ${ }^{88-96}$

\subsection{The choice of sensitization material}

Due to its large aromatic $\mathrm{sp}^{2}$ lattice, free of dangling bonds, graphene is intrinsically chemically inert. The broad sensing potential of graphene can be activated by small molecules (such as rhodamine derivatives, C8-BTBT, tetraphenyl-porphyrin, ruthenium polypyridyl), conjugated polymers, rare-earth components and even semiconductor nanoparticles (NPs) ${ }^{97-99}$ by covalent or noncovalent functionalization. Since non-covalent modification can be realized by a solution method (such as dip-coating, spincoating, and drop-casting methods and dip-pen nanolithography) and thermal evaporation (TE), it is easier than covalent modification, in which sensitive materials are attached to graphene by an amidation reaction, ${ }^{100-104}$ cycloaddition reaction, ${ }^{105-108}$ free radical addition, ${ }^{109,110}$ metal-catalysed coupling reactions ${ }^{111,112}$ and "click" chemistry. ${ }^{113-122}$ As there have been several review articles with regard to graphene functionalization, ${ }^{123-128}$ our focus herein is mainly on the non-covalent modification of small organic molecules on the surface of graphene.

Small organic molecules are an important class of materials for photodetector applications, offering wide material selection, excellent mechanical flexibility, and self-assembly or solution manufacturing capability. Recently, combining graphene with small photoactive functional components has attracted widespread attention, and several important photo-active moieties, including porphyrin, phthalocyanine, pyrenebutyrate, rhodamine 6G and so on, have been attached either to the edge or to the basal plane of the graphene surface. Hybrid nanostructures are usually synthesized by an epitaxial or solution processing method, which is suitable for large area preparation in succession with low cost. $^{129-135}$

As for small organic molecules, a solution-based procedure is a simple, yet highly efficient method for decorating a large area graphene with the lowest cost. Dip-coating, drop-casting and spin-coating are the three common solution processing methods. In the dip-coating method, graphene is directly immersed in a solution of organic molecules. First-principles density functional theory (DFT) calculations indicate that the absorption of photoactive molecules and graphene has a great relationship with dipoles, binding energy, and interfacial layer spacing between the dyes and graphene. ${ }^{136-142}$ Therefore, molecules with a high binding energy in the mixed solution have higher affinity when adsorbed with graphene, so it is difficult to control the components of organic hybridization. Compared to dip-coating, the drop-casting method can accurately control the hybrid components by direct deposition. Spin coating is one of the most common techniques for depositing thin films on substrates. It is used in a wide variety of industries and technology sectors. The advantage of spin-coating is its ability to quickly and easily produce very uniform films, ranging from a few nanometres to a few microns in thickness. The thickness and crystallinity of the active layer can be effectively adjusted by adjusting the rotation speed and concentration. Because of the simple and easy operation of the solution method, it has a good application prospect in the preparation of nanodevices.

The previously mentioned studies on organic/graphene hybrid materials mainly use the solution process without any knowledge or control of the interface quality, which could lead to severe underperformance of the phototransistors. In general, the thickness of the active layers could be well controlled with the help of CVD by tuning the evaporation temperature, growth time, and the position of the substrate. Xinran Wang and coworkers epitaxially grew C8-BTBT layers on prepatterned graphene field-effect transistors (FETs) with Au electrodes in a home-built CVD furnace to fabricate C8-BTBT/graphene hybrid organic photodetectors and studied the influence of the thickness of the active layers on the performance of devices. ${ }^{42}$

Thermal evaporation (TE) is commonly used in the deposition of electrodes. It is also a reasonable method to control the thickness of the organic layers. TE is a convenient way for follow-up processing if multiple organic layered materials are needed. Ki-Seok An et al. deposited a metal-free H2TPP/graphene hybrid layer on a substrate to fabricate a H2TPP/graphene photodetector, and then metallized the H2TPP to form ZnTPP or Al(III)TPP by using diethylzinc (DEZ) and trimethylaluminum (TMA) precursors, respectively. ${ }^{43}$

\subsection{Interaction between photoactive moieties and graphene}

Most non-covalent functionalizations have been carried out via $\pi-\pi$ stacking between conjugate structures with graphene and facilitate photo-excited charge transfer between the dye molecules and graphene, which results in the photoconductive gain. The deposition of the dye molecules can be performed using a simple dip-coating method. Using this method Jeong Ho Cho et al. prepared hybrid photodetectors by soaking a prepared graphene device in a mixture of three different dye molecules and then rinsing with deionized water. ${ }^{35}$ Interestingly, the spectra of the mixed hybrid films fabricated using this process showed only the Rh800 spectrum due to the higher affinity of Rh800 dyes to the graphene surface. However, the drop-casting and spin-coating processes enabled the adsorption of molecules (Rh101 and Rh110) having weak binding energies with graphene. The amount of sensitization molecules can be controlled through these processes, thereby adjusting the performance of the device.

\section{The progress of organic-graphene hybrid photodetectors}

\subsection{Hybrid layer prepared by the solution method}

4.1.1 Rhodamine based dye molecules. Combining graphene, which features a remarkably broad absorption band, with dye molecules (e.g., rhodamine 6G (R6G)), which have a high absorption cross-section, provides a very large photocurrent and an ultrahigh quantum efficiency. ${ }^{143-146}$ Chad A. Mirkin and coworkers patterned rhodamine 6G assembly by dip-pen nanolithography on graphene, and studied the transport and assembly properties of R6G on graphene (Fig. 2a). ${ }^{33}$ As a result, they found that, in general, the $\pi-\pi$ stacking between the aromatic 

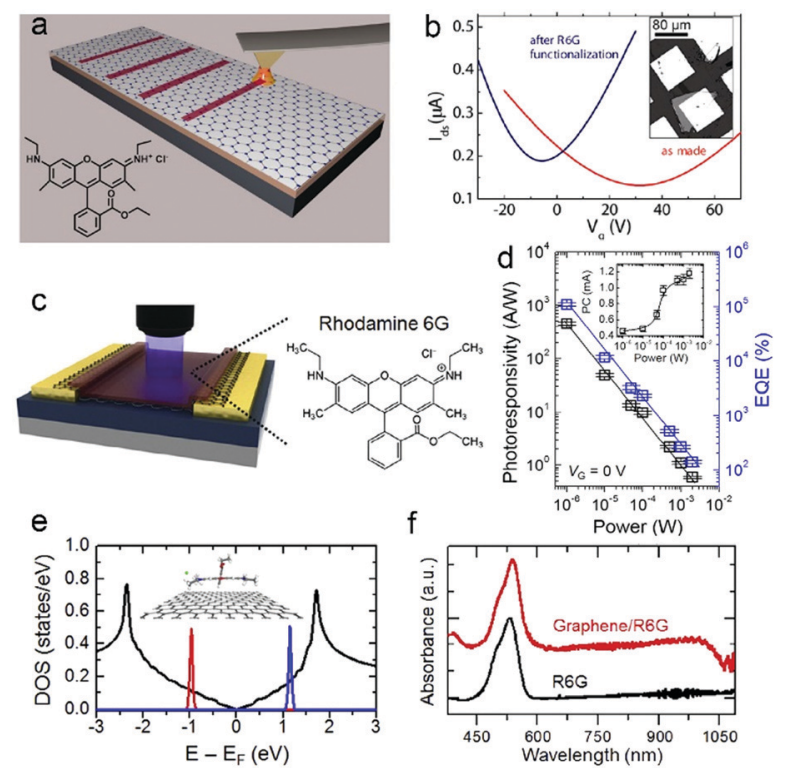

Fig. 2 (a) Schematic illustration depicting the patterning of rhodamine 6G (R6G) on graphene by dip-pen nanolithography (DPN). The inset shows the chemical structure of R6G. (b) $I_{D S}-V_{G}$ curves of the same graphene device (shown in the inset; the gold contact was prepared by evaporation of gold through a shadow mask) before (red) and after (blue) bulk functionalization with R6G. The bias voltage applied is $1 \mathrm{mV}$ for both measurements. The Dirac point moved from $\sim 32$ to $\sim-6 \mathrm{~V}$. Copyright 2013, Nano Lett. ${ }^{33}$ (c) Schematic diagram and optical microscopy top-view images of the dye sensitized graphene photodetector. (d) Responsivity $(R)$ and external quantum efficiency (EQE) as a function of the illumination power. The inset shows the photocurrent at $V_{G}=O V$ as a function of the illumination power. (e) Calculated joint density of states (DOS) of the graphene-R6G hybrid structure. The black line represents the DOS of the graphene. The positions of the HOMO (blue) and LUMO (red) levels were obtained from B3PW91 calculations of the physiosorbed R6G molecule. The HOMO of this structure was located at $-0.971 \mathrm{eV}$ and the LUMO was located at $1.150 \mathrm{eV}$ with respect to the location of the Dirac point of graphene. (f) UV-IR absorption spectra of R6G and R6G/graphene hybrid films. The red line was arbitrarily shifted upward to distinguish the absorbance of R6G from that of the R6G/graphene hybrid films. Copyright 2015, Carbon. ${ }^{34}$

components of R6G and the underlying graphene stabilizes the assembly of these molecules on the graphene surface. Moreover, the shift of the transfer curve to the direction of negative voltage suggests an n-type doping effect on graphene (Fig. 2b). This important proof-of-concept experiment suggests that it will be possible to find a series of molecules that can be used to $n$ - and p-dope graphene locally and quantitatively.

Encouraged by the above results, Jeong Ho Cho and coworkers developed a hybrid structure comprising organic dye molecules (e.g., rhodamine 6G) and graphene for the realization of highperformance optoelectronic devices (Fig. 2c). ${ }^{34}$ The dye-sensitized photodetectors respond to the incident illumination over a broad wavelength range $(400<\mathrm{k}<1000 \mathrm{~nm})$, as well as with a high responsivity $\left(\sim 460 \mathrm{~A} \mathrm{~W}^{-1}\right.$ at illumination power of $\left.1 \mu \mathrm{W}\right)$ (Fig. 2d). The photocurrent generated in the hybrid photodetector (on the order of $\mathrm{mA}$ ) is much higher than that generated in a pristine graphene photodetector (on the order of $\mu \mathrm{A}$ ). This hybrid graphene photodetector displays several advantages over other approaches: the device could operate at low voltages and the photoresponse does not vary significantly across the detector area. Apart from that, they find that both pristine R6G and the hybrid system display an absorption peak at $\sim 530 \mathrm{~nm}$ corresponding to the direct transition from the HOMO to the LUMO of R6G, which means the adsorption of R6G does not change (Fig. 2e and f). This work provides a good demonstration for designing and preparing graphene photodetectors sensitized with dye molecules to realize wavelength selective detection properties.

Based on the above work, Jeong Ho Cho and coworkers systematically investigated hybrid structures of organic dyes and graphene for the fabrication of photodetectors that display spectral color selectivity. ${ }^{35}$ Rh110, Rh101 and Rh800 with blue, green and red absorption profiles were deposited onto a graphene surface by dip-coating (Fig. 3a). The photodetectors based on the three different dye-graphene hybrid films exhibited the expected spectral color selectivities and maximum photocurrents were observed at wavelengths corresponding to the maximum absorption peak positions of each dye molecule (Fig. 3b and c). Herein, in order to broaden the spectral bandwidth over the full-color range, a resulting solution, consisting of Rh110, Rh101 and Rh800, is deposited onto a graphene channel (Fig. 3d). The mixed hybrid films exhibited a wide absorption spectral bandwidth that covered the absorption spectral ranges of each dye molecule (Fig. 3e). The mixed organic dye hybrid photodetectors offer spectral color selectivities with a high responsivity of $10^{3} \mathrm{~A} \mathrm{~W}^{-1}$ and a detectivity of $\approx 10^{10}$ Jones at an illumination power of $10 \mathrm{nW}$. The hybrid system of graphene/ organic dyes represents a versatile platform for the development of graphene-based optoelectronic devices.

4.1.2 P3HT. Conducting polymers play a key role in optoelectronics, transistors, and solar cells owing to their capability

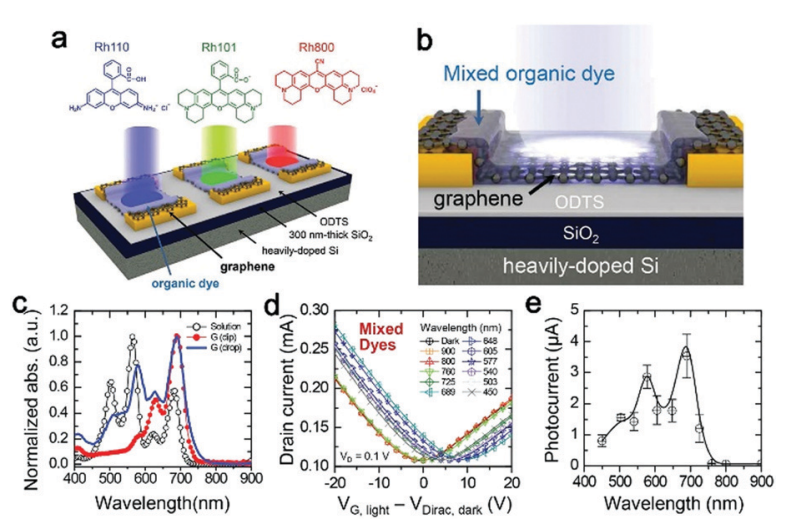

Fig. 3 (a) Schematic illustration of an organic dye-graphene hybrid photodetector based on three different rhodamine-based dye molecules (Rh110: blue, Rh 101: green, and Rh800: red). The upper panel shows the chemical structures of the organic dyes used in this study. (b) Schematic illustration of the organic dye-graphene hybrid photodetector based on the mixture of dye molecules. (c) UV-vis absorption spectra of the mixed dye solutions and films fabricated using the dip-coating and drop-casting methods. (d) ID versus $\left(V_{\mathrm{G}, \text { light }}-V_{\text {Dirac,dark }}\right)$ plots of the hybrid photodetectors based on the mixed dyes under different illumination wavelengths at a fixed incident illumination power of $1 \mu \mathrm{W}$. (e) Photocurrents at the Dirac voltages in the hybrid photodetectors. Copyright 2016, Advanced Functional Materials. ${ }^{35}$ 
a

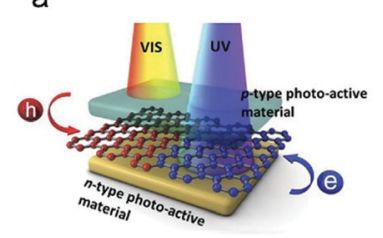

b

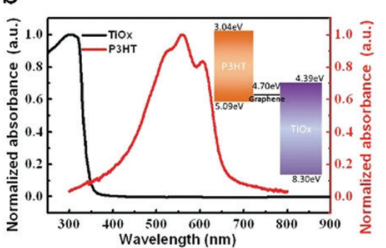

Fig. 4 (a) A proposed device structure with wavelength-selective $n$ - and p-type doping, consisting of a graphene transistor sandwiched between one UV- and one visible-light-sensitized photoactive layer. (b) The complementary UV-vis absorption spectra of the organic polymer P3HT and inorganic $\mathrm{TiO}_{x}$ thin films of the OIHD platform. The inset shows the corresponding energy levels of graphene based on the OIHD platform. Copyright 2015, Advanced Materials. ${ }^{36}$

in charge carrier transport. ${ }^{147-154}$ P3HT, which has been widely used in polymer solar cell devices, typically absorbs visible light between 350 and $700 \mathrm{~nm}$, and transports holes. ${ }^{155,156}$ Most sensitized graphene photoelectronic devices reported in the literature have typically demonstrated single-type carrier transport under light illumination (either n- or p-type), where the charge-transfer process is predominantly determined by the energy band alignment between graphene and the lightabsorbing material. Hence, it will be particularly attractive if the carrier transport types (either n- or p-type) of a sensitized graphene transistor can be further controlled using selective optical excitation wavelengths, resulting in tuneable carrier transport in a graphene-based photoelectronic device. Chunwei Chen and coworkers proposed a novel device structure consisting of a graphene transistor sandwiched between two photoactive layers. ${ }^{36}$ In this phototransistor, the organic polymer poly(3hexylthiophene) (P3HT) and inorganic $\mathrm{TiO}_{x}$ (amorphous $\mathrm{TiO}_{2}$ ) thin films act as the top and bottom doping layers, respectively, which is shown in Fig. 4a. The band alignments of the corresponding energy levels of the organic/inorganic hybrid doping (OIHD) platform, shown in the inset of Fig. 4b, suggests that the two opposite carriers might be able to transfer from the two photoactive layers to graphene when the device is under selective UV or visible light illumination. Experimental results show that the OIHD platform offers a unique advantage to provide controllable and reversible n-type or p-type doping on graphene by using selective-wavelength illumination. The concept of this novel device structure thus enables the control of the dual carrier-type transport behaviors of a graphene transistor by using wavelength-selective illumination, which may have great potential in future development of optically controlled electronic devices or integrated circuits.

4.1.3 Graphene-ruthenium complex hybrid photodetectors. In energy conversion applications such as solar cells and water splitting, ruthenium polypyridyl complexes have been used as photoactive components for graphene or carbon nanotube (CNT)based nanocomposites. ${ }^{157-160}$ These hybrid materials are known to facilitate separation and transport of electron-hole pairs, and reduce the recombination of charge carriers. These results have attracted researchers to exploit transition metal complexes as charge transfer materials in photodetectors. Joon Hak Oh and coworkers fabricated graphene-based photodetectors with a

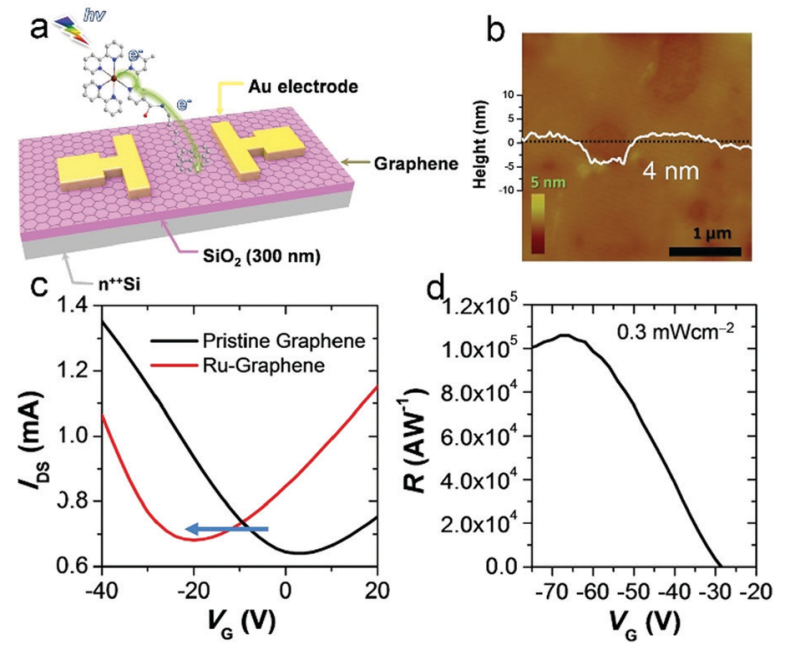

Fig. 5 (a) Schematic depiction of a graphene-ruthenium complex hybrid FET-type photodetector. In the chemical structure of complex 1, H atoms were omitted and $\mathrm{C}, \mathrm{N}, \mathrm{O}$, and $\mathrm{Ru}$ atoms are represented by gray, blue, light-red, and dark-red balls. For clarity, the scale of complex 1 is enlarged compared to graphene. (b) AFM height and surface profile (white line) along the black dotted line (ruthenium complex $1 /$ graphene $/ \mathrm{SiO}_{2} / \mathrm{Si}$ ). (c) Transfer characteristics of pristine graphene and ruthenium complex $1 /$ graphene devices $\left(V_{D S}=1 \mathrm{~V}\right)$ under dark conditions. (d) Responsivity as a function of gate voltage (the wavelength of incident light: $\lambda=450 \mathrm{~nm}$ ). Copyright 2014, Small. ${ }^{37}$

$4 \mathrm{~nm}$-thick ruthenium complex hybrid (Fig. 5a and b). ${ }^{37}$ The monolayer graphene-based photodetectors exhibit pronounced n-type doping effect due to electron transfer via the metalligand charge transfer (MLCT) from the ruthenium complex to graphene, and shows an ultrahigh responsivity of $\approx 1 \times$ $10^{5} \mathrm{~A} \mathrm{~W}^{-1}$ and a photoconductive gain of $\approx 3 \times 10^{6}$ under incident optical intensity of the order of sub-milliwatts (Fig. 5c and d). This methodology opens a viable way for enhancing the photoresponsivity of graphene-based FET-type photodetectors, and can be extended to construct various state-of-the-art photodetector platforms that are based on $\pi$-conjugated photoactive materials including organic small molecules, polymers, and carbon nanotubes.

4.1.4 Structure switching molecule: spiropyran. Photochromic molecules, which can switch between two (meta-) stable states when exposed to specific wavelengths, enable the use of a photonic input to modulate the electrical characteristics of solid-state devices. Spiropyran (SP, the uncharged, parent closed-ring isomer), a wellknown photochromic molecule, comprises an indoline and a chromene moiety bound together via a spiro junction and oriented perpendicular with respect to one another (MC, the planar, active, highly conjugated structure). ${ }^{161}$ The spiropyran-merocyanine system can be switched between two stable states when exposed to specific wavelengths (ultraviolet light from SP to MC structure, and visible light for the reverse process), which has the potential for use as a photonic input to modulate the electrical characteristics of solid-state devices. ${ }^{162-174}$ Of particular interest is the possibility of exploiting photochromic molecules to modulate the conductance of (semi)conductive materials, eventually leading to light-switchable macroscopic devices. This approach was 


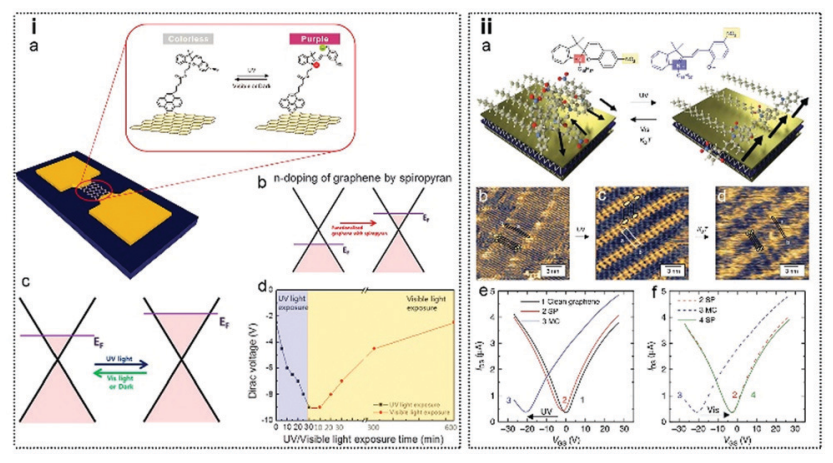

Fig. 6 (i-a) Schematic illustration of a FET device with spiropyran functionalized graphene. (b) Energy level change of graphene after being functionalized with spiropyran. (c) Energy level change upon UV and visible light exposure. (d) Dirac point change as a function of UV and visible light exposure time. Copyright 2012, ACS Nano. ${ }^{38}$ (ii-a) Photo-switchable molecular crystals in two dimensions. A schematic representation of the approach. A spiropyran (SP) derivative forms ordered crystalline structures when deposited on different van der Waals substrates. In the cartoon, a $\mathrm{MoS}_{2}$ single layer is depicted in which the yellow (blue) layer represents the S-(Mo-) atomic plane. Photoinduced isomerization induces a structural rearrangement. The molecular dipoles (depicted as black arrows) are randomly oriented before irradiation, yet well aligned after UV irradiation, leading to a modification in the energetics of the van der Waals substrate. The chemical structure of the spiropyran (SP)-merocyanine (MC) derivative used in the study is also shown. (b-d) Scanning tunneling microscopic imaging of SP assemblies on HOPG. Height images of the molecular assemblies obtained (b) after spin-coating the SP solution, (c) immediately after UV irradiation, and (d) $48 \mathrm{~h}$ after UV irradiation. A schematic sketch of the molecule is superimposed onto the images to facilitate the visualization of the molecular ordering. Tunneling parameters: $I_{\mathrm{t}}=20 \mathrm{pA}\left(\mathrm{b}, \mathrm{c}\right.$ and d), $V_{\mathrm{t}}=1000 \mathrm{mV}(\mathrm{b}$ and d), $600 \mathrm{mV}$ (c). Electrical characteristics of devices, (e) trace 1 (black): clean graphene, trace 2 (red): graphene covered with the SP layer, trace 3 (blue): graphene/MC after UV irradiation over the whole flake. The arrow highlights the negative shift in the threshold voltage accompanying the $\mathrm{SP} \rightarrow \mathrm{MC}$ isomerization, indicative of n-type doping. (f) Trace 4 (green): recovered graphene/SP after green light irradiation of the whole graphene surface. Traces 2 and 3 are replotted for clarity (dashed). Copyright Nature communication, 2018. ${ }^{39}$

demonstrated for carbon nanotubes, graphene and polymers. Hyeon Suk Shin and coworkers designed and synthesized pyrene-SP molecules, and then demonstrated defect-free n-doping of graphene through functionalization with SP by noncovalent bonding (Fig. 6i-a and b). ${ }^{38}$ The conformational change between SP and MC upon UV and visible light irradiation affected the electrical properties of graphene. ${ }^{175}$ They demonstrated a reversible light-modulated Dirac point of graphene according to UV and visible light exposure (Fig. 6i-c and d). This allows for new possibilities of doping control methods using functionalization of graphene with photochromic molecules.

At present, in the study of SP molecules, the isomerization of molecular structure is inferred based on electrical properties, without a direct, real-space visualization of the (supra)molecular structural changes induced by the switching events. However, scanning tunneling microscopy (STM) is a good way to acquire the real-space images of supramolecular assemblies of photochromic molecules. Paolo Samorì and coworkers achieved exquisite control over events taking place from the molecular level to the device scale by combining different experimental and theoretical approaches (Fig. 6ii-a). ${ }^{39}$ They designed and synthesized a spiropyran (SP) derivative bearing an 18-carbon long alkyl chain, and monitored the evolution of the molecular arrangement with sub-nm resolution by STM imaging in air and at room temperature on dry films on HOPG to study the photoswitch of the SP derivative down to the monolayer limit. As for the STM image, photochromic molecules assembled at the surface of single layer graphene generate an atomically precise superlattice in which a major structural rearrangement is obtained by light-induced collective isomerization (Fig. 6ii-b-d). As a result, the rearrangement causes a reversible shift in the work function of two-dimensional materials (2DMs), readable in devices as significant doping, which is also fully reversible (Fig. 6ii-e and f). This work offers a yet unexplored solution to supramolecular electronics, in which atomic precision in molecular self-assembly is tailored not to optimize charge transport but rather to control it by imparting new properties to a material, enabling the realization of multifunctional, high-performance devices.

4.1.5 Single-walled carbon nanotube (SWCNTs). Singlewalled carbon nanotubes (SWCNTs) are considered as one of the most promising candidates for next-generation photoelectronic devices due to their high carrier mobility, high thermal conductivity, large absorption coefficient, and excellent chemical stability. ${ }^{176,177}$ The bandgaps of semiconducting SWCNTs are inversely proportional to their diameter (from 0.5 to $1.2 \mathrm{eV}$ when the diameters range from 0.7 to $2.0 \mathrm{~nm}),{ }^{178}$ with which the wavelength of SWCNT-based photodetectors can be tuned by controlling the diameters. In addition, it has been theoretically and experimentally demonstrated that multiple electron-hole pairs can be generated from one high-energy photon in SWCNTs, which provides the possibility of fabricating high-performance photoelectronic devices using different nanotube types as a light absorber, such as individual SWCNTs, aligned SWCNT arrays, SWCNT networks, SWCNT films or even hybrid materials (such as SWCNT/C 60 ). ${ }^{179}$ In addition to 0D C60, 2D graphene has also been demonstrated to enhance significantly the carrier separation by forming Schottky junctions with SWCNTs due to exceedingly high carrier mobility and thermal conductivity. ${ }^{180,181}$ However, the intrinsic large dark current of graphene and the poor optical absorption of the ultrathin SWCNT layer inhibit further improvement in the detectivity and on-off ratio of the devices. Zhang et al. demonstrate a carbon-based photodetector using SWCNT/separated graphene (SGR) hybrid networks, which is illustrated in Fig. 7a and b. ${ }^{40}$ In this hybrid material, graphene nanosheets were connected by SWCNTs with the dip-coating method to form a continuous network. The discontinuous graphene nanosheets exhibit a much lower dark current than the SWCNT/graphene film, which enhances the current to a large extent. Moreover, the efficient charge transfer between SWCNTs and SGR nanosheets can significantly reduce the recombination probability for photogenerated electrons and holes, thus enhancing the photoresponsivity of the photodetector. As for the result, the device exhibits an excellent broadband photoelectric response from the visible to the NIR range (405$1064 \mathrm{~nm}$ ) with a high photoresponsivity of $>3000 \mathrm{~A} \mathrm{~W}^{-1}$ and a fast response time of $44 \mu \mathrm{s}$, which is higher than that of other 

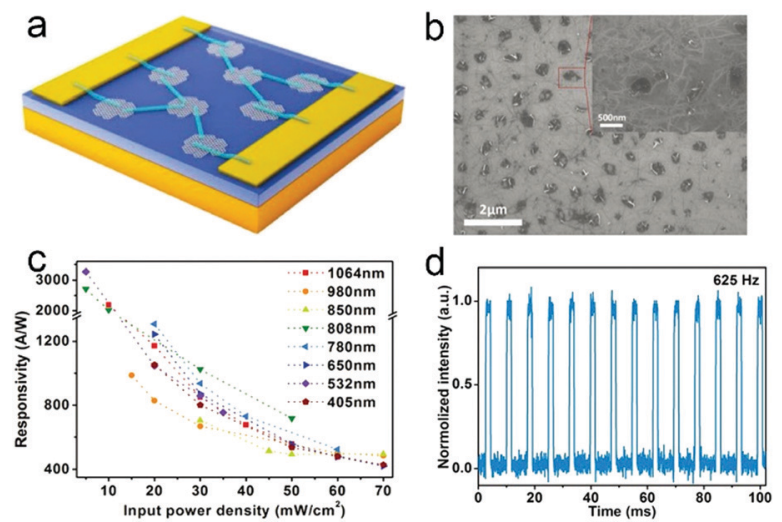

Fig. 7 (a) Schematic illustration of a carbon-based photodetector (b) Typical SEM images of SWCNT/SGR hybrid networks in the channel. (c) Responsivity as a function of incident power density operating at $10 \mathrm{~V}$ under different illumination wavelengths. (d) Transient photoresponse of the device to pulsed illumination $\left(1064 \mathrm{~nm}, 35 \mathrm{~mW} \mathrm{~cm}{ }^{-2}\right)$ with a frequency of $625 \mathrm{~Hz}$. Copyright Advanced Optical Materials 2018 . $^{40}$

SWCNT-based devices (Fig. 7c and d). This architecture strategy not only demonstrates great potential for high-performance all carbon photodetectors but also offers a universal approach to fabricate high-performance semiconductor/graphene hybrid photodetectors.

4.1.6 Biomaterials: cytochrome $c$. van der Waals heterojunctions that interface graphene with functional biomolecules can provide a unique scheme for biomolecule-based circuits on a large scale. High-performance optoelectronics through atomicscale interface design can provide high-efficiency exciton dissociation and charge transfer as demonstrated in inorganic van der Waals heterojunctions consisting of semiconductor nanostructures on graphene or other 2D materials. ${ }^{182-191}$ A biomolecule/ graphene van der Waals heterojunction provides a generic platform for designing high-performance, flexible, and scalable optoelectronics. A key challenge in controllable attachment is the formation of a desired interfacial electronic structure by the biomolecules for a high-efficiency optoelectronic process involving photoabsorption, exciton dissociation, carrier transfer, and transport. In Wu's work, the modification of graphene with a well characterized protein molecule cytochrome $c$ (Cyt $c$ ) was achieved by the print method on the channel of graphene field effect transistors (GFETs) (Fig. 8a). ${ }^{41}$ Benefitting from the electrostatic field in graphene, the orientation of the Cyt $c$ molecules can be controlled on the polarity-controlled GFET channel with a desired interfacial electronic structure for exciton dissociation and charge transfer by the built-in electric field established through the band-edge alignment at the Cyt $c$ /GFET interface (Fig. 8b and c). This Cyt c/GFET van der Waals heterojunction photodetector combines the photoabsorption of biomolecule Cyt $c$ with the superior carrier mobility of graphene, enabling a high-efficiency optoelectronic process as illustrated in the high photoconductive gain of up to $1.1 \times 10^{5}$ and the high responsivity of $1.39 \times 10^{4} \mathrm{~A} \mathrm{~W}^{-1}$ at $340 \mathrm{~nm}$ and $7.57 \times 10^{4} \mathrm{~A} \mathrm{~W}^{-1}$ at $410 \mathrm{~nm}$ (Fig. 8d). In addition, the biomolecule/graphene van der Waals heterojunction nanohybrid structure integrates the spectral
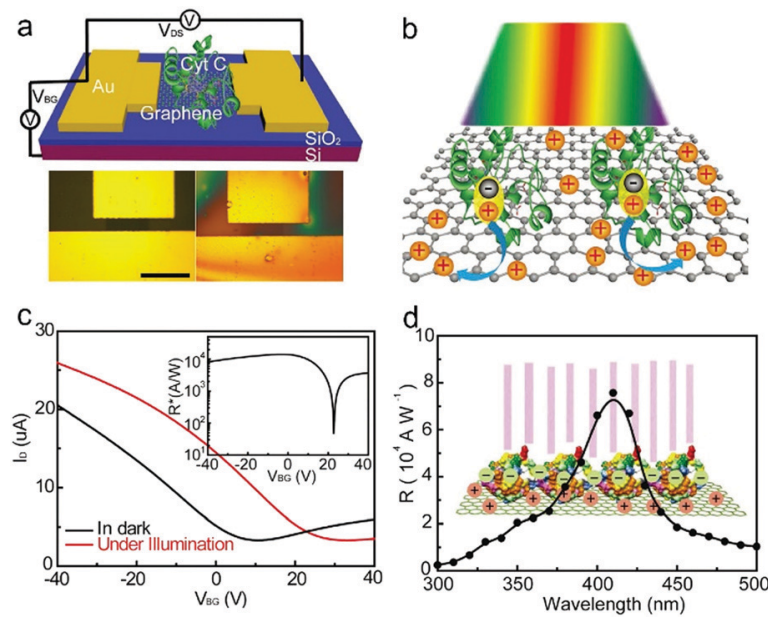

Fig. 8 (a) Schematic image of the Cyt c/GFET heterojunction photodetector (upper). Image of a GFET channel before and after the Cyt $c$ was printed on its channel, respectively (the scale bar is $5 \mu \mathrm{m}$ ). (b) Schematic description of the Cyt $c$ molecules absorbing the photons under illumination and the generated excitons separated by the built-in electric field due to the band-edge alignment at the Cyt c/graphene interface. (c) I $I_{\mathrm{D}}$ versus $V_{\mathrm{BG}}$ curves of a Cyt c/GFET heterojunction photodetector (in vacuum) measured in the dark and under illumination (340 nm, power density of $1.34 \mathrm{~mW} \mathrm{~cm}^{-2}$ ). Inset: Calculated photoresponsivity as a function of $V_{B G}$. (d) Spectral photoresponsivity of the Cyt C/GFET heterojunction photodetectors. Inset: Scheme of interface charges after exciton dissociation. Copyright Advanced Functional Materials, $2018 .^{41}$

tunability of biomolecules for photoabsorption, GFET gate tunability of the interfacial band-edge alignment for optimal exciton dissociation and charge transfer, and high carrier mobility of graphene for charge transport. The approach of printable biomolecules on GFETs will pave a way for larger-scale fabrication of low-cost, high-performance nanohybrid optoelectronic devices.

\subsection{Hybrid layer prepared by self-assembly}

4.2.1 Physical vapor deposition (PVD) for ultrathin organic crystals. Organic field effect transistors (OFETs) attract much attention for next generation devices. To achieve a good performance of OFETs, improvements of crystallinity and control of the molecular orientation of the active material are essential. For this purpose, Xinran Wang et al. have epitaxially grown C8-BTBT layers on prepatterned graphene field-effect transistors (FETs) with Au electrodes to fabricate an ultrathin epitaxial organic crystal/graphene hybrid for highly efficient phototransistors (Fig. 9a). ${ }^{42}$ The thickness of the C8-BTBT layer could be effectively controlled by this method (Fig. 9b). Benefitting from the high quality of organic crystals and interfaces, which is a unique feature of vdW epitaxy (van der Waals epitaxy), the phototransistors exhibit a strong photoresponse down to the limit of monolayer organic semiconductors, with responsivity higher than $10^{4} \mathrm{~A} \mathrm{~W}^{-1}$, response time of $\approx 25 \mathrm{~ms}$, and photoconductive gain over $10^{8}$. The roughly linear relationship of EQE and gain with film thickness up to approximately seven layers suggested that excitons in the top C8-BTBT layers could still diffuse to the interface and dissociate efficiently, implying an exciton diffusion length of at least $\approx 20 \mathrm{~nm}$. Due to the exponentially 

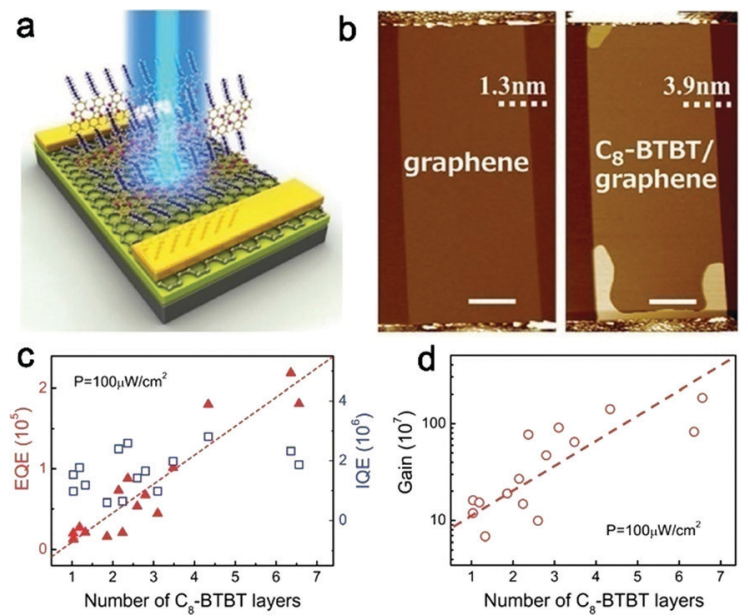

Fig. 9 (a) Schematic illustration of a typical C8-BTBT-graphene phototransistor. (b) The AFM images of a graphene FET before (left) and after (right) C8-BTBT epitaxial growth. The device is mostly covered with IL $+1 \mathrm{~L}$ C8-BTBT as reflected from the measured height increase by $2.6 \mathrm{~nm}$. Scale bars: $2 \mu \mathrm{m}$. (c) EQE (red triangles) and IQE (blue squares) as a function of average number of C8-BTBT layers for all the measured devices (laser power, $100 \mu \mathrm{W} \mathrm{cm}{ }^{-2}$ ). The red dashed line reflects the linear increase of EQE. (d) Photoconductive gain $\left(G_{\mathrm{ph}}\right)$ as a function of average number of C8-BTBT layers for all the measured devices (laser power, $100 \mu \mathrm{W} \mathrm{cm}{ }^{-2}$ ). The red dashed line reflects the exponential increase of $G_{\mathrm{ph}}$. Copyright 2016, Advanced Materials. ${ }^{42}$

longer lifetime in thicker samples, they observed an exponential increase of $G_{\mathrm{ph}}$ as a function of C8-BTBT thickness, despite the large scatter of data points (presumably, because the number of layers does not reflect the film morphology) (Fig. 9b and c). Given the large library of organic molecules, they believe that epitaxial ultrathin organic crystals on graphene can serve as a versatile platform for high-performance, broadband phototransistors.

4.2.2 Thermal evaporation (TE) for tetraphenyl-porphyrin and metalloporphyrin. Thermal evaporation (TE) is a simple and easy approach for the controllable fabrication of photodetectors based on graphene-organic hybrid thin films. In Ki-Seok An and coworkers' work, metal-free $\mathrm{H}_{2}$ TPP or metalloporphyrins (Al(III)TPP and ZnTPP) are synthesized by thermal evaporation and vapor phase metalation which are utilized for tunable functionalization of graphene-based devices. ${ }^{43}$ As a proof of concept, they successfully fabricated various graphene-based photodetectors via simple and efficient vapor-phase metalation of porphyrin, as shown in Fig. 10a-d. The photoresponsivities of H2TPP-, ZnTPP-, and Al(III)TPP-graphene photodetectors are $0.22 \mathrm{~A} \mathrm{~W}^{-1}, 0.54 \mathrm{~A} \mathrm{~W}^{-1}$, and $5.36 \mathrm{~A} \mathrm{~W}^{-1}$, respectively, at a $50 \mathrm{mV}$ bias voltage (Fig. 10e and f). It should be noted that the devised synthesis method provides a high degree of flexibility in the fabrication of graphene-based electronics. As such, the research described herein may allow for the realization of advanced flexible and transparent graphene devices (e.g., thin film transistors and memory devices).

4.2.3 Self-assembly of D-A type molecule. Nature employs self-assembly to fabricate the most complex molecularly precise machinery known to man. Bottom-up modular self-assembled networks and 2D materials grant access to device fabrication

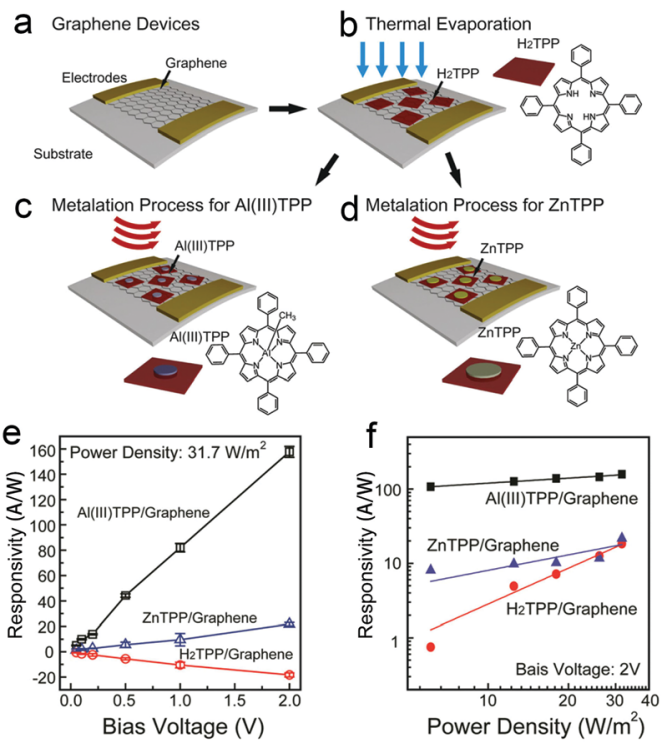

Fig. 10 (a-d) Schematic representation of H2TPP- and metalloporphyringraphene hybrid film formation. (e) Photoresponsivity of H2TPP-, ZnTPP-, and Al(III)TPP-graphene-based devices as a function of the source-drain bias voltage during exposure to light with a power density of $31.7 \mathrm{~W} \mathrm{~m}^{-2}$. (f) Photoresponsivity as a function of the light power density; a bias voltage of 2 V was used for the measurements. Copyright 2016, Nanotechnology. ${ }^{43}$

with molecular precision. ${ }^{192}$ Heteromolecular, two-dimensional self-assembled networks provide a route to spatially organize different building blocks relative to each other, enabling molecularly precise fabrication. ${ }^{193,194}$ Sarah Wieghold and coworkers fabricated devices with molecular precision by a near-to-monolayer architecture on graphene, which consist of a self-assembled terrylene-based dye and melamine (Fig. 11a and b). ${ }^{44}$ The molecular architecture of the self-assembled sensitizing layer approaches atomically defined spatial disposition of all components. In this work, CVD graphene is successfully transferred onto a transparent diamond, and terrylene diimide (TDI) derivative (1) and melamine (2) which with a chromophore are self-assembled as a network on the surface of graphene. The assembly at the graphene-diamond interface shows an absorption maximum at $740 \mathrm{~nm}$ (Fig. 11c), whereby the photoresponse can be measured with a gallium counter electrode. Employing $19 \mathrm{~mW} \mathrm{~cm}{ }^{-2}$ irradiation intensity at $710 \mathrm{~nm}$, the photocurrent and opencircuit voltage are $0.5 \mathrm{nA}$ and $270 \mathrm{mV}$, respectively (Fig. 11d). By estimation of the tunnelling area, their non-optimized device element configuration yields an incident photon to electron efficiency (IPCE) (at $710 \mathrm{~nm}$ ) as high as $0.6 \%$ in air, opening novel avenues towards tandem photovoltaics from monolayerthin sensitizers. What's more, this work motivates rapid progress in molecular engineered manufacturing and monolayer by monolayer molecular printing methods, which potentially grants access to exponential optimization of device performance.

In addition, Thomas P. Russell and coworkers alternately installed typical triphenylamine (TPA) as an electron-rich unit and BTTh $_{2}$ (4,7-bisthienyl-2,1,3-benzothiadiazole) as an electron-deficient unit into acetylene linked conjugated macrocycles (Fig. 12). ${ }^{45}$ STM experiments confirmed that the two shape-persistent 

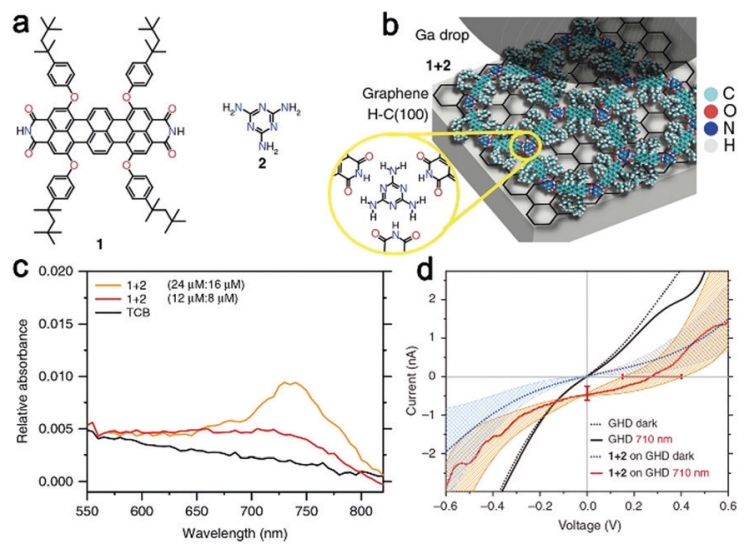

Fig. 11 (a) Structure of TDI tetracarboxylic acid derivative (1) and melamine (2). (b) Schematic drawing of the device setup including the ideal representation of the $1+2$ mixture yielding a hexagonal supramolecular network via hydrogen bonds (yellow circle). (c) $1+2$ (12 mM: $8 \mathrm{mM}$ ) and pure 1,2,4-trichlorobenzene (TCB) baseline absorbance spectra. The result with the concentrations of molecules 1 and 2 increased to $24 \mathrm{mM}: 16 \mathrm{mM}$ is also shown as additional evidence of strong absorbance reduction upon complexation with 2. (d) Current-voltage characteristics before (black lines) and after (green lines) $\lambda=520 \mathrm{~nm}$ photon irradiation. Approach parameters: $I_{\mathrm{t}}=2 \mathrm{nA}$ and $V_{\mathrm{t}}=100 \mathrm{mV}$. Striped areas indicate the maximum and minimum currents observed in more than ten curves in a single junction, while error bars indicate the standard deviation for five junctions in three samples and are reported in the main text as $I_{\mathrm{SC}}=(0.5 \pm 0.2) \mathrm{nA}$, $V_{\mathrm{OC}}=(270 \pm 120) \mathrm{mV}$. Copyright 2016, Nature Communications. ${ }^{44}$

cyclic molecules showed interesting concentration-controlled self-assembling behaviour at the solid/liquid interface and could capture fullerenes within their inner cavities to form host-guest coassembly structures. Moreover, in the new stable complexes, D-A conjugated cyclic molecules act as donors and fullerene $\left(\mathrm{C}_{70}\right)$ as an acceptor to form new donor-acceptor heterojunctions with big shape complementarity D/A molecular interfaces. More importantly, this work provides further opportunities to achieve high-efficiency organic solar cells through utilizing D-A conjugated cyclic molecules as donor materials with PCBM to control the morphology of the active layer.

\subsection{Covalent organic frameworks (COFs)}

Covalent organic frameworks (COFs), consisting of molecular building blocks connected by covalent bonds, are a new class of porous architectures that allow the integration of organic units with atomic precision into long-range-ordered two- or threedimensional structures. ${ }^{195-203}$ Owing to the covalent linkage of the components, as well as the elaborately controlled structure, including the nature of the structural unit molecule, COFs are promising for photodetectors. Theoretical calculations have predicted the attractive properties of $2 \mathrm{D}$ conjugated polymers for more than two decades. ${ }^{204}$ In fact, the Jiang group has already explored the photoelectric properties of bulk COFs in recent years. They show that metallophthalocyanine, porphyrin and its self-assembly materials have great potential in photoelectric devices. ${ }^{205-207}$ However, the poor dispersibility of bulk COFs is the major drawback in their optoelectronic applications.

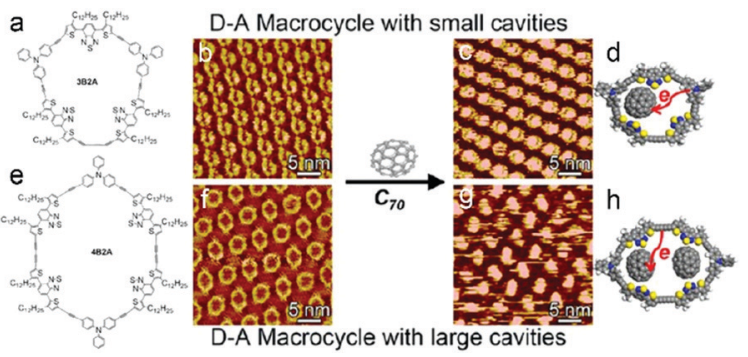

Fig. 12 ( $a$ and e) The chemical structure of two conjugated D-A macrocycles. (b and f) STM image of the molecular self-assembly structure at the HOPG/1-phenyloctane interface under certain solution concentrations. (c and g) STM images of the coassembly structure of 3B2A and 4B2A with $\mathrm{C}_{70}$ respectively at the HOPG/1-phenyloctane interface and their corresponding calculated models (d and h). Copyright ACS Nano, 2017.45

2D COFs with layered structures stacked together through van der Waals interactions are ideally suited for efficient energy storage and charge transport. The surface confined synthesis on single-crystal surfaces is a simple while efficient methodology for the preparation of 2D COFs with single layer thickness, and easy control of the number of layers, as well as the bandgap. Lei and coworkers designed a simple while efficient methodology for the preparation of $2 \mathrm{D} \mathrm{CO}_{\mathrm{FBTA}-\mathrm{PDA}}$ at an octanoic acid/graphene interface. $^{46}$ As the synthesized $2 \mathrm{D}$ COF film is adsorbed on graphene, there is a strong coupling between surface $\mathrm{COF}_{\mathrm{BTA}-\mathrm{PDA}}$ and graphene, which was revealed by the contrast of surface $\mathrm{COF}_{\mathrm{BTA}-\mathrm{PDA}}$ on different parts of the graphene surface (Fig. 13a and $b$ ). The shape of the density of states (DOS) of the G-surface $\mathrm{COF}_{\mathrm{BTA}-\mathrm{PDA}}$ near the Fermi level is dominated by graphene and

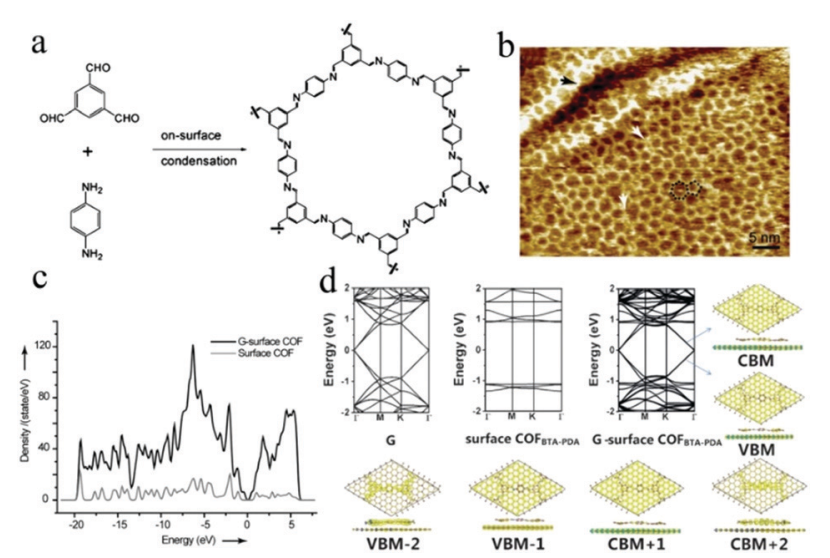

Fig. 13 (a) Reaction scheme of Schiff base condensation between benzene-1,3,5-tricarbaldehyde (BTA) and $p$-phenylenediamine (PDA) into hexagonal 2D surface COF BTA-PDA. (b) STM image of surface COF $_{B T A-P D A}$ on SLG-copper (single layer graphene grown by CVD on copper). A " $5+7$ " defect is highlighted by an overlaid schematic model and two typical domain boundaries are marked by the white arrows. A perfect chain of hexagons crossing the step edge of copper is marked by a black arrow. (c) Density of states of $\mathrm{G}$-surface $\mathrm{COF}_{\mathrm{BTA}-\mathrm{PDA}}$ (the surface of $\mathrm{COF}_{\mathrm{BTA} A \mathrm{PDA}}{ }^{-}$ graphene complex) and surface $\mathrm{COF}_{\mathrm{BTA} \text {-PDA }}$ (Fermi level is set at $0 \mathrm{eV}$ ). (d) The band structures of graphene, surface COF COTA-PDA, G-surface COF $_{\text {BTA-PDA }}$ and the decomposed charge density of G-surface $\mathrm{COF}_{\mathrm{BTA} \text {-PDA }}$ near the Fermi level (Fermi level is set at $0 \mathrm{eV}$ ). Copyright 2014, Angewandte Chemie International Edition. ${ }^{46}$ 
the band gap of the G-surface $\mathrm{COF}_{\mathrm{BTA}-\mathrm{PDA}}$ complex is very small $(0.081 \mathrm{eV})$, which indicate that the imine based surface COFs are intriguing two dimensional organic semiconductors with a tuneable band gap (Fig. 13c and d). It provides a potential technological approach to sensitize graphene with 2D COFs with controllable thickness for applications in flexible devices.

\section{Conclusion and perspectives}

Graphene, a single layer 2D structure, possesses excellent physical and chemical properties, such as high mobility and thermal conductivity, great mechanical strength and extremely large specific surface area. However, pristine graphene shows a limited application in photodetectors because of its low optical absorption and zero band gap. In order to improve the performance of graphene-based photodetectors, a series of methods have been used to achieve high-quality photodetection, including both device geometry and materials engineering.

Over the past few decades, the booming of studies on developing chemically functionalized graphene created great opportunities for the development of hybrid thin film transistors, and numerous dramatic and unprecedented results are demonstrated in front of the world, including many excellent results using photo-active moieties to functionalize graphene. In this article, we have systematically introduced the application of graphene functionalized with organic molecules in photodetectors in three progressive parts: the gain mechanism in optical gain, the integration of device and the progress in applications of hybrid films in photodetectors. Based on these contents, it is worth noting that the aforementioned graphene hybrid nanostructures will play an important role in improving the performance and extending the function of traditional photodetectors.

Although many successful examples have been demonstrated, large-scale, practical applications of graphene-based hybrid materials in photodetection and photodetectors still have a long way to go. Furthermore, in order to achieve the preparation of a graphene-based photodetector with a large area, stable performance and commercial application, there are still some problems that should be optimized. Firstly, how to produce high-quality, large-area, low-defect (structure defects or oxidation sites) graphene or graphene sheets is still a challenge in the preparation and improvement of photodetectors and to go into the mechanism of graphene-based photodetectors. Therefore, new methodologies for milder exfoliation and chemical modification of graphene are still needed to tackle this problem. Secondly, since graphene cannot directly generate long-lived charge carriers ${ }^{208}$ in photoelectron light energy conversion applications, it is necessary to make more carefully designs of chemical functionalization and linking groups, in order to take advantage of the electronic interaction between graphene and photoactive groups. ${ }^{209,210}$ Multi-functionalized graphene materials with different small organic molecules are expected to provide new ideas for designing charge transfer systems for light energy conversion. Moreover, with the development of nanotechnology, integrated nanosystems and multifunction circuits have attracted much research attention due to their potential applications in many fields. Thus, the development of integratable nanophotodetectors is highly needed.

For many types of detectors, performance parameters have already been demonstrated at levels that are superior or on a par with existing technologies. To move forward, the ultrahigh photoresponsivity or ultrafast response speed of graphene-based photodetectors is still a challenge to have them both in a single device. This is a prerequisite for graphene-based photodectors to realize commercialization. In addition, the prospect of commercialization depends not only on the performance of the detectors, but also on their unique strengths and capabilities, as well as the ability to achieve large-scale, high-quality GRM production at low cost, and the creation of large-energy and long-lived photons and integration of electronic platforms such as CMOS technology.

\section{Conflicts of interest}

There are no conflicts to declare.

\section{Acknowledgements}

This work was supported by the National Science Foundation of China $(21572157,21872103,51633006)$ and the Ministry of Science and Technology of China (Grants 2016YFB0401100).

\section{Notes and references}

1 E. H. Hwang, S. Adam and S. Das Sarma, Phys. Rev. Lett., 2007, 98, 186806.

2 F. Schedin, A. K. Geim, S. V. Morozov, E. W. Hill, P. Blake, M. I. Katsnelson and K. S. Novoselov, Nat. Mater., 2007, 6, 652.

3 R. R. Nair, P. Blake, A. N. Grigorenko, K. S. Novoselov, T. J. Booth, T. Stauber, N. M. R. Peres and A. K. Geim, Science, 2008, 320, 1308.

4 S. Pang, Y. Hernandez, X. Feng and K. Müllen, Adv. Mater., 2011, 23, 2779.

5 K. Nomura and A. H. MacDonald, Phys. Rev. Lett., 2006, 96, 256602.

6 C. M. Weber, D. M. Eisele, J. P. Rabe, Y. Liang, X. Feng, L. Zhi, K. Müllen, J. L. Lyon, R. Williams, D. A. V. Bout and K. J. Stevenson, Small, 2010, 6, 184.

7 M. L. Müeller, X. Yan, B. Dragnea and L. Li, Nano Lett., 2011, 11, 56.

8 P. Plochocka, P. Kossacki, A. Golnik, T. Kazimierczuk, C. Berger, W. A. de Heer and M. Potemski, Phys. Rev. B: Condens. Matter Mater. Phys., 2009, 80, 245415.

9 Y. Yang and R. Murali, Appl. Phys. Lett., 2011, 98, 093116. 10 K. K. Manga, J. Wang, M. Lin, J. Zhang, M. Nesladek, V. Nalla, W. Ji and K. P. Loh, Adv. Mater., 2012, 24, 1697.

11 S. Ryu, L. Liu, S. Berciaud, Y. Yu, H. Liu, P. Kim, G. W. Flynn and L. E. Brus, Nano Lett., 2010, 10, 4944.

12 H. Xu, Y. Chen, J. Zhang and H. Zhang, Small, 2012, 8, 2833.

13 F. Schedin, A. K. Geim, S. V. Morozov, E. W. Hill, P. Blake, M. I. Katsnelson and K. S. Novoselov, Nat. Mater., 2007, 6, 652. 
14 X. Dong, D. Fu, W. Fang, Y. Shi, P. Chen and L.-J. Li, Small, 2009, 5, 1422.

15 A. K. Geim, Science, 2009, 324, 1530.

16 W. Wei and X. Qu, Small, 2012, 8, 2138.

17 I. Gierz, C. Riedl, U. Starke, C. R. Ast and K. Kern, Nano Lett., 2008, 8, 4603.

18 K. S. Novoselov, A. K. Geim, S. V. Morozov, D. Jiang, Y. Zhang, S. V. Dubonos, I. V. Grigorieva and A. A. Firsov, Science, 2004, 306, 666.

19 P. Plochocka, P. Kossacki, A. Golnik, T. Kazimierczuk, C. Berger, W. A. de Heer and M. Potemski, Phys. Rev. B: Condens. Matter Mater. Phys., 2009, 80, 245415.

20 K. K. Manga, J. Wang, M. Lin, J. Zhang, M. Nesladek, V. Nalla, W. Ji and K. P. Loh, Adv. Mater., 2012, 24, 1697.

21 H. Xu, Y. Chen, J. Zhang and H. Zhang, Small, 2012, 8, 2833.

22 M. L. Müeller, X. Yan, B. Dragnea and L. Li, Nano Lett., 2011, 11, 56.

23 M. Gobbi, E. Orgiu and P. Samorì, Adv. Mater., 2018, 30, 1706103.

24 H. Geng, D. Yuan, Z. Yang, Z. Tang, X. Zhang, K. Yang and Y. Su, J. Mater. Chem. C, 2019, 7, 11056.

25 W. J. Yu, Y. Liu, H. Zhou, A. Yin, Z. Li, Y. Huang and X. Duan, Nat. Nanotechnol., 2013, 8, 952-958.

26 H. Tan, Y. Fan, Y. Zhou, Q. Chen, W. Xu and J. H. Warner, ACS Nano, 2016, 10, 7866-7873.

27 L. Britnell, R. M. Ribeiro, A. Eckmann, R. Jalil, B. D. Belle, A. Mishchenko, Y.-J. Kim, R. V. Gorbachev, T. Georgiou, S. V. Morozov, A. N. Grigorenko, A. K. Geim, C. Casiraghi, A. H. Castro Neto and K. S. Novoselov, Science, 2013, 340, 1311-1314.

28 Z. Sun, Z. Liu, J. Li, G. Tai, S. Lau and F. Yan, Adv. Mater., 2012, 24, 5878-5883.

29 G. Konstantatos, M. Badioli, L. Gaudreau, J. Osmond, M. Bernechea, F. P. G. Arquer, F. Gatti and F. H. L. Koppens, Nat. Nanotechnol., 2012, 7, 363-368.

30 Z. Xia, P. Li, Y. Wang, T. Song, Q. Zhang and B. Sun, ACS Appl. Mater. Interfaces, 2015, 7, 24136-24141.

31 C. Teng, D. Xie, M. Sun, S. Chen, P. Yang and Y. Sun, ACS Appl. Mater. Interfaces, 2016, 8, 31289-31294.

32 Z. Tan, Y. Wu, H. Hong, J. Yin, J. Zhang, L. Lin, M. Wang, X. Sun, L. Sun, Y. Huang, K. Liu, Z. Liu and H. Peng, J. Am. Chem. Soc., 2016, 138, 16612-16615.

33 G. Zhou, S. He, K. A. Brown, J. Mendez-Arroyo, F. Boey and C. A. Mirkin, Nano Lett., 2013, 13, 1616-1621.

34 Y. Lee, S. Yu, J. Jeon, H. Kim, J. Y. Lee, H. Kim, J. H. Ahn, E. Hwang and J. H. Cho, Carbon, 2015, 88, 165-172.

35 Y. S. Gim, Y. Lee, S. Kim, S. Hao, M. S. Kang, W. J. Yoo, H. Kim, C. Wolverton and J. Ho Cho, Adv. Funct. Mater., 2016, 26, 6593-6600.

36 P. Ho, S. Li, Y. Liou, C. Wen, Y. Chung and C. Chen, Adv. Mater., 2015, 27, 282-287.

37 X. Liu, E. K. Lee and J. H. Oh, Small, 2014, 10, 3700-3706.

38 A. Jang, E. Jeon, D. Kang, G. Kim, B. S. Kim, D. J. Kang and H. S. Shin, ACS Nano, 2012, 6, 9207-9213.

39 M. Gobbi, S. Bonacchi, J. Lian, A. Vercouter, S. Bertolazzi, B. Zyska, M. Timpel, R. Tatti, Y. Olivier, S. Hecht, M. V. Nardi,
D. Beljonne, E. Orgiu and P. Samori, Nat. Commun., 2018, 9, 2661.

40 B. Cai, Y. Su, Z. Tao, J. Hu, C. Zou, Z. Yang and Y. Zhang, Adv. Opt. Mater., 2018, 6, 1800791.

41 M. Gong, P. Adhikari, Y. Gong, T. Wang, Q. Liu, B. Kattel, W. Ching, W. Chan and J. Wu, Adv. Funct. Mater., 2018, 28, 1704797.

42 X. Liu, X. Luo, H. Nan, H. Guo, P. Wang, L. Zhang, M. Zhou, Z. Yang, Y. Shi, W. Hu, Z. Ni, T. Qiu, Z. Yu, J. Xu and X. Wang, Adv. Mater., 2016, 28, 5200-5205.

43 S. J. Kim, W. Song, S. Kim, M. S. Myung, S. S. Lee, J. Lim and K. An, Nanotechnology, 2016, 27, 075709.

44 S. Wieghold, J. Li, P. Simon, M. Krause, Y. Avlasevich, C. Li, J. A. Garrido, U. Heiz, P. Samorì, K. Müllen, F. Esch, J. V. Barth and C. A. Palma, Nat. Commun., 2016, 7, 10700.

45 S. Zhang, Z. Liu, W. Fu, F. Liu, C. Wang, C. Sheng, Y. Wang, K. Deng, Q. Zeng, L. Shu, J. Wan, H. Chen and T. Russell, ACS Nano, 2017, 11, 11701-11713.

46 L. Xu, X. Zhou, W. Tian, T. Gao, Y. Zhang, S. Lei and Z. Liu, Angew. Chem., Int. Ed., 2014, 53, 9564-9568.

47 A. H. Castro Neto, F. Guinea, N. M. R. Peres, K. S. Novoselov and A. K. Geim, Rev. Mod. Phys., 2009, 81, 109.

48 F. Bonaccorso, Z. Sun, T. Hasan and A. C. Ferrari, Nat. Photonics, 2010, 4, 611.

49 K. S. Kim, Y. Zhao, H. Jang, S. Y. Lee, J. M. Kim, J. H. Ahn, P. Kim, J. Y. Choi and B. H. Hong, Nature, 2009, 457, 706.

50 F. Xia, T. Mueller, Y. Lin, A. V. Garcia and P. Avouris, Nat. Nanotechnol., 2009, 4, 839.

51 M. Breusing, C. Ropers and T. Elsaesser, Phys. Rev. Lett., 2009, 102, 086809.

52 G. Konstantatos, M. Badioli, L. Gaudreau, J. Osmond, M. Bernechea, F. P. G. de Arquer, F. Gatti and F. H. L. Koppens, Nat. Nanotechnol., 2012, 7, 363-368.

53 T. Ohta, A. Bostwick, T. Seyller, K. Horn and E. Rotenberg, Science, 2006, 313, 951-954.

54 H. Chang, J. Cheng, X. Liu, J. Gao, M. Li, J. Li, X. Tao, F. Ding and Z. Zheng, Chem. - Eur. J., 2011, 17, 8896-8903.

55 S. H. Yu, Y. Lee, S. K. Jang, J. Kang, J. Jeon, C. Lee, J. Y. Lee, H. Kim, E. Hwang and S. Lee, ACS Nano, 2014, 8, 8285.

56 A. Rose, Concepts in Photoconductivity and Allied Problems, Robert E. Krieger Publishing, 1978.

57 C. Wang, X. Ren, C. Xu, B. Fu, R. Wang, X. Zhang, R. Li, H. Li, H. Dong, Y. Zhen, S. Lei, L. Jiang and W. Hu, Adv. Mater., 2018, 30, 1706260.

58 Y. Wu, P. Liu, B. S. Ong, T. Srikumar, N. Zhao, G. Botton and S. Zhu, Appl. Phys. Lett., 2005, 86, 142102.

59 M. Shkunov, R. Simms, M. Heeney, S. Tierney and I. McCulloch, Adv. Mater., 2005, 17, 2608-2612.

60 K. Nakayama, Y. Hirose, J. Soeda, M. Yoshizumi, T. Uemura, M. Uno, W. Li, M. J. Kang, M. Yamagishi, Y. Okada, E. Miyazaki, Y. Nakazawa, A. Nakao, K. Takimiya and J. Takeya, Adv. Mater., 2011, 23, 1626-1629.

61 Y. Liu, K. He, G. Chen, W. R. Leow and X. Chen, Chem. Rev., 2017, 117, 12893-12941.

62 Z. Liu, J. Xu, D. Chen and G. Shen, Chem. Soc. Rev., 2015, 44, 161-192. 
63 D. J. Lipomi, Adv. Mater., 2016, 28, 4180-4183.

64 J. Pu, Y. Yomogida, K. Liu, L. Li, Y. Iwasa and T. Takenobu, Nano Lett., 2012, 12, 4013-4017.

65 H. Yang, W. R. Leow and X. Chen, Small Methods, 2018, 2, 1700259.

66 Y. Zhang, T. Tang, C. Girit, Z. Hao, M. Martin, A. Zettl, M. Crommie, Y. Shen and F. Wang, Nature, 2009, 459, 820-823.

67 Z. Li, E. A. Henriksen, Z. Jiang, Z. Hao, M. C. Martin, P. Kim, H. Stormer and D. N. Basov, Nat. Phys., 2008, 4, 532-535.

68 F. Wang, Y. Zhang, C. Tian, C. Girit, A. Zettl, M. Crommie and Y. Shen, Science, 2008, 320, 206-209.

69 W. Knap, V. Kachorovskii, Y. Deng, S. Rumyantsev, J. Lu, R. Gaska, M. S. Shur, G. Simin, X. Hu, M. A. Khan, C. A. Saylor and L. C. Brunel, J. Appl. Phys., 2002, 91, 9346.

70 G. Konstantatos, M. Badioli, L. Gaudreau, J. Osmond, M. Bernechea, F. P. G. de Arquer, F. Gatti and F. H. L. Koppens, Nat. Nanotechnol., 2012, 7, 363-368.

71 K. Roy, M. Padmanabhan, S. Goswami, T. P. Sai, G. Ramalingam, S. Raghavan and A. Ghosh, Nat. Nanotechnol., 2013, 8, 826-830.

72 L. Prechtel, L. Song, D. Schuh, P. Ajayan, W. Wegscheider and A. Holleitner, Nat. Commun., 2012, 3, 646.

73 M. Freitag, T. Low and P. Avouris, Nano Lett., 2013, 13, 1644-1648.

74 E. Stern, J. F. Klemic, D. A. Routenberg, P. N. Wyrembak, D. B. Turner-Evans, A. D. Hamilton, D. A. LaVan, T. M. Fahmy and M. A. Reed, Nature, 2007, 445, 519.

75 A. Reina, X. Jia, J. Ho, D. Nezich, H. Son, V. Bulovic, M. S. Dresselhaus and J. Kong, Nano Lett., 2009, 9, 30-35.

76 X. S. Li, W. Cai, J. H. An, S. Kim, J. Nah, D. Yang, R. Piner, A. Velamakanni, I. Jung, E. Tutuc, S. K. Banerjee, L. Colombo and R. S. Ruoff, Science, 2009, 324, 1312-1314.

77 L. Liu, H. Zhou, R. Cheng, Y. Chen, Y. Lin, Y. Qu, J. Bai, I. A. Ivanov, G. Liu, G. Y. Huang and X. Duan, J. Mater. Chem., 2012, 22, 1498-1503.

78 H. Zhou, W. Yu, L. Liu, R. Cheng, Y. Chen, X. Huang, Y. Liu, Y. Wang, Y. Huang and X. Duan, Nat. Commun., 2013, 4, 2096.

79 B. Radisavljevic, A. Radenovic, J. Brivio, V. Giacometti and A. Kis, Nat. Nanotechnol., 2011, 6, 147-150.

80 X. Cai, A. B. Sushkov, R. J. Suess, M. M. Jadidi, G. S. Jenkins, L. O. Nyakiti, R. L. Myers-Ward, S. Li, J. Yan, D. K. Gaskill, T. E. Murphy, H. D. Drew and M. S. Fuhrer, Nat. Nanotechnol., 2014, 9, 814-819.

81 N. M. Gabor, J. C. W. Song, Q. Ma, N. L. Nair, T. Taychatanapat, K. Watanabe, T. Taniguchi, L. S. Levitov and P. Jarillo-Herrero, Science, 2011, 334, 648-652.

82 E. J. H. Lee, K. Balasubramanian, R. T. Weitz, M. Burghard and K. Kern, Nat. Nanotechnol., 2008, 3, 486-490.

83 M. Freitag, T. Low and P. Avouris, Nano Lett., 2013, 13, 1644-1648.

84 K. Parvez, Z. Wu, R. Li, X. Liu, R. Graf, X. Feng and K. Müllen, J. Am. Chem. Soc., 2014, 136, 6083-6091.

85 K. S. Kim, Y. Zhao, H. Jang, S. Y. Lee, J. M. Kim, K. S. Kim, J. H. Ahn, P. Kim, J. Y. Choi and B. H. Hong, Nature, 2009, 457-706.
86 X. Li, W. Cai, J. An, S. Kim, J. Nah, D. Yang, R. Piner, A. Velamakanni, I. Jung, E. Tutuc, S. K. Banerjee, L. Colombo and R. S. Ruoff, Science, 2009, 324, 1312.

87 X. Li, C. W. Magnuson, A. Venugopal, R. M. Tromp, J. B. Hannon, E. M. Vogel, L. Colombo and R. S. Ruoff, J. Am. Chem. Soc., 2011, 133, 2816.

88 Z. Sun, Z. Yan, J. Yao, E. Beitler, Y. Zhu and J. M. Tour, Nature, 2010, 468, 549.

89 A. Guermoune, T. Chari, F. Popescu, S. S. Sabri, J. Guillemette, H. S. Skulason, T. Szkopek and M. Siaj, Carbon, 2011, 49, 4204.

90 Y. Gamo, A. Nagashima, M. Wakabayashi, M. Terai and C. Oshima, Surf. Sci., 1997, 374, 61.

91 H. Ueta, M. Saida, C. Nakai, Y. Yamada, M. Sasaki and S. Yamamoto, Surf. Sci., 2004, 560, 183.

92 S. Marchini, S. Günther and J. Wintterlin, Phys. Rev. B: Condens. Matter Mater. Phys., 2007, 76, 075429.

93 J. Coraux, A. T. N'Diaye, C. Busse and T. Michely, Nano Lett. , 2008, 8, 565.

94 R. S. Edwards and K. S. Coleman, Acc. Chem. Res., 2012, 46, 23.

95 S. Bae, H. Kim, Y. Lee, X. Xu, J. S. Park, Y. Zheng, J. Balakrishnan, T. Lei, H. R. Kim, Y. Song, Y. J. Kim, K. S. Kim, B. Ozyilmaz, J. H. Ahn, B. Hong and S. Iijima, Nat. Nanotechnol., 2010, 5, 574.

96 Z. Sun, Z. Liu, J. Li, G. Tai, S. Lau and F. Yan, Adv. Mater., 2012, 24, 5878-5883.

97 G. Konstantatos, M. Badioli, L. Gaudreau, J. Osmond, M. Bernechea, F. Pelayo Garcia de Arquer, F. Gatti and F. H. L. Koppens, Nat. Nanotechnol., 2012, 7, 363-368.

98 C. Chiang, G. Haider, W. Tan, Y. Liou, Y. Lai, R. Ravindranath, H. Chang and Y. Chen, ACS Appl. Mater. Interfaces, 2016, 8, 466-471.

99 N. Karousis, A. S. D. Sandanayaka, T. Hasobe, S. P. Economopoulos, E. Sarantopoulou and N. Tagmatarchis, J. Mater. Chem., 2011, 21, 109.

100 Y. Xu, Z. Liu, X. Zhang, Y. Wang, J. Tian, Y. Huang, Y. Ma, X. Zhang and Y. Chen, Adv. Mater., 2009, 21, 1275.

101 N. Karousis, J. Ortiz, K. Ohkubo, T. Hasobe, S. Fukuzumi, Á. Sastre-Santos and N. Tagmatarchis, J. Phys. Chem. C, 2012, 116, 20564.

102 J. Zhu, Y. Li, Y. Chen, J. Wang, B. Zhang, J. Zhang and W. J. Blau, Carbon, 2011, 49, 1900.

103 X. Zhang, X. Zhao, Z. Liu, Y. Liu, Y. Chen and J. Tian, Opt. Express, 2009, 17, 23959.

104 X. Zhang, L. Hou, A. Cnossen, A. C. Coleman, O. Ivashenko, P. Rudolf, B. J. van Wees, W. R. Browne and B. L. Feringa, Chem. - Eur. J., 2011, 17, 8957.

105 M. E. Ragoussi, J. Malig, G. Katsukis, B. Butz, E. Spiecker, G. de la Torre, T. Torres and D. M. Guldi, Angew. Chem., Int. Ed., 2012, 51, 6421.

106 F. M. Koehler and W. J. Stark, Acc. Chem. Res., 2013, 46(10), 2297-2306.

107 M. Quintana, E. Vazquez and M. Prato, Acc. Chem. Res., 2013, 46, 138.

108 E. Bekyarova, S. Sarkar, F. Wang, M. E. Itkis, I. Kalinina, X. Tian and R. C. Haddon, Acc. Chem. Res., 2013, 46, 65. 
109 J. R. Lomeda, C. D. Doyle, D. V. Kosynkin, W. F. Hwang and J. M. Tour, J. Am. Chem. Soc., 2008, 130, 16201.

110 T. Umeyama, J. Mihara, N. Tezuka, Y. Matano, K. Stranius, V. Chukharev, N. V. Tkachenko, H. Lemmetyinen, K. Noda, K. Matsushige, T. Shishido, Z. Liu, K. Hirose-Takai, K. Suenaga and H. Imahori, Chem. - Eur. J., 2012, 18, 4250.

111 Q. Bao, H. Zhang, J. Yang, S. Wang, D. Y. Tang, R. Jose, S. Ramakrishna, C. T. Lim and K. P. Loh, Adv. Funct. Mater., 2010, 20, 782.

112 H. C. Kolb, M. G. Finn and K. B. Sharpless, Angew. Chem., Int. Ed., 2001, 40, 2004.

113 X. Xu, J. Chen, X. Luo, J. Lu, H. Zhou, W. Wu, H. Zhan, Y. Dong, S. Yan, J. Qin and Z. Li, Chem. - Eur. J., 2012, 18, 14384.

114 M. Castelaín, G. Martínez, P. Merino, J. Á. Martín-Gago, J. L. Segura, G. Ellis and H. J. Salavagione, Chem. - Eur. J., 2012, 18, 4965.

115 X. Chen, A. B. Braunschweig, M. J. Wiester, S. Yeganeh, M. A. Ratner and C. A. Mirkin, Angew. Chem., Int. Ed., 2009, 48, 5178.

116 H. Wang, K. Zhou, Y. Xie, J. Zeng, N. Chai, J. Li and H. L. Zhang, Chem. Commun., 2011, 47, 5747.

117 J. Iehl, R. P. de Freitas and J.-F. Nierengarten, Tetrahedron Lett., 2008, 49, 4063.

118 V. V. Rostovtsev, L. G. Green, V. V. Fokin and K. B. Sharpless, Angew. Chem., Int. Ed., 2002, 41, 2596.

119 S. Campidelli, B. Ballesteros, A. Filoramo, D. D. Diaz, G. de la Torre, T. Torres, G. M. A. Rahman, C. Ehli, D. Kiessling, F. Werner, V. Sgobba, D. M. Guldi, C. Cioffi, M. Prato and J.-P. Bourgoin, J. Am. Chem. Soc., 2008, 130, 11503.

120 H. Li, F. Cheng, A. M. Duft and A. Adronov, J. Am. Chem. Soc., 2005, 127, 14518.

121 T. Palacin, H. L. Khanh, B. Jousselme, P. Jegou, A. Filoramo, C. Ehli, D. M. Guldi and S. P. Campidelli, J. Am. Chem. Soc., 2009, 131, 15394.

122 D. R. Dreyer, S. Park, C. W. Bielawski and R. S. Ruoff, Chem. Soc. Rev., 2010, 39, 228.

123 K. P. Loh, Q. Bao, P. K. Ang and J. Yang, J. Mater. Chem., 2010, 20, 2277.

124 Z. Sun and H. Chang, ACS Nano, 2014, 8, 4133-4156.

125 H. Wang, Q. Wang, K. Zhou and H. Zhang, Small, 2013, 9, 1266-1283.

126 J. Li, L. Niu, Z. Zheng and F. Yan, Adv. Mater., 2014, 26, 5239-5273.

127 M. Quintana, E. Vazquez and M. Prato, Acc. Chem. Res., 2013, 46, 138.

128 P. Liu, Y. Wu, Y. Li, B. Ong and S. Zhu, J. Am. Chem. Soc., 2006, 128, 4554.

129 K. Manga, S. Wang, M. Jaiswal, Q. Bao and K. P. Loh, Adv. Mater., 2010, 22, 5265-5270.

130 T. Wakahara, P. D’Angelo, K. I. Miyazawa, Y. Nemoto, O. Ito, N. Tanigaki, D. D. C. Bradley and T. D. Anthopoulos, J. Am. Chem. Soc., 2012, 134, 7204-7206.

131 Y. Wang, Y. Li, W. Zhu, J. Liu, X. Zhang, R. Li, Y. Zhen, H. Dong and W. Hu, Nanoscale, 2016, 8, 14920-14924.

132 J. Lu, C. Xu, J. Dai, J. Li, Y. Wang, Y. Lin and P. Li, Nanoscale, 2015, 7, 3396.
133 K. Liu, M. Sakurai, M. Liao and M. Aono, J. Phys. Chem. C, 2010, 114, 19835.

134 L. Hu, L. Wu, M. Liao, X. Hu and X. Fang, Adv. Funct. Mater., 2012, 22, 998.

135 O. Leenaerts, B. Partoens and F. Peeters, Phys. Rev. B: Condens. Matter Mater. Phys., 2009, 79, 235440.

136 E. K. Gross and R. M. Dreizler, Density Functional Theory, Springer Science \& Business Media, New York, 2013, vol. 337.

137 G. Kresse and D. Joubert, Phys. Rev. B: Condens. Matter Mater. Phys., 1999, 59, 1758.

138 G. Kresse and J. Hafner, Phys. Rev. B: Condens. Matter Mater. Phys., 1993, 47, 558.

139 H. J. Monkhorst and J. D. Pack, Phys. Rev. B: Solid State, 1976, 13, 5188.

140 X. Liu, X. Luo, H. Nan, H. Guo, P. Wang, L. Zhang, M. Zhou, Z. Yang, Y. Shi, W. Hu, Z. Ni, T. Qiu, Z. Yu, J. Xu and X. Wang, Adv. Mater., 2016, 28, 5200-5205.

141 X. Chen, X. Liu, B. Wu, H. Nan, H. Guo, Z. Ni, F. Wang, X. Wang, Y. Shi and X. Wang, Nano Lett., 2017, 17, 6391.

142 L. Gao, C. Ge, W. Li, C. Jia, K. Zeng, W. Pan, H. Wu, Y. Zhao, Y. He, J. He, Z. Zhao, G. Niu, X. Guo, F. P. G. de Arquer, E. H. Sargent and J. Tang, Adv. Funct. Mater., 2017, 27, 1702360.

143 T. Zhang, Z. Li, J. Wang, W. Kong, G. Wu, Y. Zheng, Y. Zhao, E. Yao, N. Zhuang and L. Luo, Sci. Rep., 2016, 6, 38569 .

144 K. Sekiguchi, S. Yamaguchi and T. Tahara, J. Phys. Chem. A, 2006, 110, 2601.

145 X. Liu, X. Chen, J. Yi, Z. Luo, H. Nan, H. Guo, Z. Ni, Y. Ding, S. Dai and X. Wang, Org. Electron., 2019, 64, 22.

146 D. H. Kim, J. T. Han, Y. D. Park, Y. Jang, J. H. Cho, M. Hwang and K. Cho, Adv. Mater., 2006, 18, 719-723.

147 J. N. Wilson, W. Steffen, T. G. McKenzie, G. Lieser, M. Oda, D. Neher and U. H. F. Bunz, J. Am. Chem. Soc., 2002, 124, 6830-6831.

148 H. Dong, S. Jiang, L. Jiang, Y. Liu, H. Li, W. Hu, E. Wang, S. Yan, Z. Wei, W. Xu and X. Gong, J. Am. Chem. Soc., 2009, 131, 17315-17320.

149 H. A. Um, D. H. Lee, D. U. Heo, D. S. Yang, J. Shin, H. Baik, M. J. Cho and D. H. Choi, ACS Nano, 2015, 9, 5264-5274.

150 Y. Liu, H. Dong, S. Jiang, G. Zhao, Q. Shi, J. Tan, L. Jiang, W. Hu and X. Zhan, Chem. Mater., 2013, 25, 2649-2655.

151 S. Wang, M. Kappl, I. Liebewirth, M. Müller, K. Kirchhoff, W. Pisula and K. Müllen, Adv. Mater., 2012, 24, 417-420.

152 Y. Yao, H. Dong, F. Liu, T. P. Russell and W. Hu, Adv. Mater., 2017, 29, 1701251.

153 C. Liu, Q. Wang, H. Tian, Y. Geng and D. Yan, Polymer, 2013, 54, 2459-2465.

154 K. M. Coakley and M. D. McGehee, Chem. Mater., 2004, 16, 4533.

155 W. Ma, C. Yang, X. Gong, K. Lee and A. J. Heeger, Adv. Funct. Mater., 2005, 15, 1617.

156 J. Zhang, J. Yu, M. Jaroniec and J. Gong, Nano Lett., 2012, 12,4584 .

157 Z. K. Liu, J. H. Li, Z. H. Sun, G. A. Tai, S. P. Lau and F. Yan, ACS Nano, 2012, 6, 810. 
158 A. Mukherji, B. Seger, G. Lu and L. Wang, ACS Nano, 2011, 5, 3483.

159 F. Li, B. Zhang, X. Li, Y. Jiang, L. Chen, Y. Li and L. Sun, Angew. Chem., Int. Ed., 2011, 50, 12276.

160 B. L. Feringa and W. R. Browne, Molecular Switches, WileyVCH, Weinheim, 2011.

161 A. C. Whalley, M. L. Steigerwald, X. Guo and C. Nuckolls, J. Am. Chem. Soc., 2017, 129, 12590-12591.

162 J. M. Mativetsky, G. Pace, M. Elbing, M. A. Rampi, M. Mayor and P. Samori, J. Am. Chem. Soc., 2008, 130, 9192-9193.

163 A. J. Kronemeijer, H. B. Akkerman, T. Kudernac, B. J. van Wees, B. L. Feringa and P. W. M. Blom, Adv. Mater., 2008, 20, 1467-1473.

164 E. Margapoti, P. Strobel, M. M. Asmar, M. Seifert, J. Li, M. Sachsenhauser, O. Ceylan, C. A. Palma, J. V. Barth, J. A. Garrido, A. Cattani-Scholz, S. E. Ulloa and J. J. Finley, Nano Lett., 2014, 14, 6823-6827.

165 E. Margapoti, J. Li, O. Ceylan, M. Seifert, F. Nisic, T. L. Anh, F. Meggendorfer, C. Dragonetti, C. A. Palma, J. V. Barth and J. J. Finley, Adv. Mater., 2015, 27, 1426-1431.

166 C. Jia, A. Migliore, N. Xin, S. Huang, J. Wang, Q. Yang, S. Wang, H. Chen, D. Wang, B. Feng, Z. Liu, G. Zhang, D. Qu, H. Tian, M. A. Ratner, H. Xu, A. Nitzan and X. Guo, Science, 2016, 352, 1443-1446.

167 X. Guo, L. Huang, S. O'Brien, P. Kim and C. Nuckolls, J. Am. Chem. Soc., 2005, 127, 15045-15047.

168 J. M. Simmons, I. in, V. E. Campbell, T. J. Mark, F. Léonard, P. Gopalan and M. A. Eriksson, Phys. Rev. Lett., 2007, 98, 86802.

169 M. Kim, N. S. Safron, C. Huang, M. S. Arnold and P. Gopalan, Nano Lett., 2012, 12, 182-187.

170 A. Jang, E. K. Jeon, D. Kang, G. Kim, B. S. Kim, D. Kang and H. Shin, ACS Nano, 2012, 6, 9207-9213.

171 E. Orgiu, N. Crivillers, M. Herder, L. Grubert, M. Patzel, J. Frisch, E. Pavlica, D. T. Duong, G. Bratina, A. Salleo, N. Koch, S. Hecht and P. Samori, Nat. Chem., 2012, 4, 675-679.

172 E. Orgiu and P. Samorì, Adv. Mater., 2014, 26, 1827-1844.

173 M. Suda, R. Kato and H. M. Yamamoto, Science, 2015, 347, 743-746.

174 L. Yang, S. Wang, Q. Zeng, Z. Zhang and L. Peng, Small, 2013, 9, 1225.

175 X. He, F. Léonard and J. Kono, Adv. Opt. Mater., 2015, 3, 989.

176 T. Lei, I. Pochorovski and Z. Bao, Acc. Chem. Res., 2017, 50, 1096.

177 M. Gao, Z. Huang, B. Zeng, T. Pan, Y. Zhang, H. Peng and Y. Lin, Appl. Phys. Lett., 2015, 106, 051601.

178 C. Liu, Y. Chang, T. B. Norris and Z. Zhong, Nat. Nanotechnol., 2014, 9, 273.

179 A. D. Bartolomeo, Phys. Rep., 2016, 606, 1.

180 B. Cai, Y. Su, Z. Tao, J. Hu, C. Zou, Z. Yang and Y. Zhang, Adv. Opt. Mater., 2018, 6, 1800791.

181 Y. Deng, Z. Luo, N. J. Conrad, H. Liu, Y. Gong, S. Najmaei, P. M. Ajayan, J. Luo, X. Xu and P. Ye, Nano Lett., 2005, 8, 8292.

182 D. Jariwala, T. J. Marks and M. C. Hersam, Nat. Mater., 2017, 16, 170.

183 W. Xia, L. Dai, P. Yu, X. Tong, W. Song, G. Zhang and Z. Wang, Nanoscale, 2017, 9, 4324.
184 J. He, N. Kumar, M. Z. Bellus, H. Y. Chiu, D. He, Y. Wang and H. Zhao, Nat. Commun., 2014, 5, 5622.

185 J. He, D. He, Y. Wang and H. Zhao, Opt. Express, 2017, 25, 1949.

186 M. Gong, Q. Liu, R. Goul, D. Ewing, M. Casper, A. Stramel, A. Elliot and J. Wu, ACS Appl. Mater. Interfaces, 2017, 9, 27801.

187 Q. Liu, B. Cook, M. Gong, Y. Gong, D. Ewing, M. Casper, A. Stramel and J. Wu, ACS Appl. Mater. Interfaces, 2017, 9, 12728.

188 P. Wang, S. Liu, W. Luo, H. Fang, F. Gong, N. Guo, Z. Chen, J. Zou, Y. Huang, X. Zhou, J. Wang, X. Chen, W. Lu, F. Xiu and W. Hu, Adv. Mater., 2017, 29, 1604439.

189 C. Xie, C. Mak, X. Tao and F. Yan, Adv. Funct. Mater., 2017, 27, 1603886.

190 H. Cheng, G. Wang, D. Li, Q. He, A. Yin, Y. Liu, H. Wu, M. Ding, Y. Huang and X. Duan, Nano Lett., 2016, 16, 367.

191 C. A. Palma and P. Samorì, Blueprinting macromolecular electronics, Nat. Chem., 2011, 3, 431-436.

192 J. A. Theobald, N. S. Oxtoby, M. A. Phillips, N. R. Champness and P. H. Beton, Nature, 2013, 424, 1029-1031.

193 J. A. Zerkowski, C. T. Seto and G. M. Whitesides, J. Am. Chem. Soc., 1992, 114, 5473-5475.

194 B. Zhang, M. T. Trinh, B. Fowler, M. Ball, Q. Xu, F. Ng, M. L. Steigerwald, X. Zhu, C. Nuckolls and Y. Zhong, J. Am. Chem. Soc., 2016, 138, 16426-16431.

195 A. Thomas, Angew. Chem., 2010, 122, 8506-8523.

196 A. Thomas, Angew. Chem., Int. Ed., 2010, 49, 8328-8344.

197 X. Feng, X. Ding and D. Jiang, Chem. Soc. Rev., 2012, 41, 6010-6022.

198 A. J. Doonan, D. J. Tranchemontagne, T. G. Glover, J. R. Hunt and O. M. Yaghi, Nat. Chem., 2010, 2, 235-238.

199 N. L. Campbell, R. Clowes, L. K. Ritchie and A. I. Cooper, Chem. Mater., 2009, 21, 204-206.

200 H. Furukawa and O. M. Yaghi, J. Am. Chem. Soc., 2009, 131, 8875-8883.

201 Y. G. Zhang and S. N. Riduan, Chem. Soc. Rev., 2012, 41, 2083-2094.

202 S. Wan, J. Guo, J. Kim, H. Ihee and D. Jiang, Angew. Chem., Int. Ed., 2009, 48, 5439-5442.

203 S. Wan, J. Guo, J. Kim, H. Ihee and D. Jiang, Angew. Chem., 2009, 121, 5547-5550.

204 G. Kresse and J. Furthmuller, Phys. Rev. B: Condens. Matter Mater. Phys., 1996, 54, 11169-11186.

205 X. Feng, L. Liu, Y. Honsho, A. Saeki, S. Seki, S. Irle, Y. Dong, A. Nagai and D. Jiang, Angew. Chem., Int. Ed., 2012, 51, 2618-2622.

206 X. Ding, J. Guo, X. Feng, Y. Honsho, J. Guo, S. Seki, P. Maitarad, A. Saeki, S. Nagase and D. Jiang, Angew. Chem., Int. Ed., 2011, 50, 1289-1293.

207 X. Chen, M. Addicoat, E. Jin, L. Zhai, H. Xu, N. Huang, Z. Guo, L. Liu, S. Irle and D. Jiang, J. Am. Chem. Soc., 2015, 137, 3241-3247.

208 P. V. Kamat, J. Phys. Chem. Lett., 2011, 2, 242.

209 S. Zhu, S. Tang, J. Zhang and B. Yang, Chem. Commun., 2012, 48, 4527.

210 X. Lv, Y. Huang, Z. Liu, J. Tian, Y. Wang, Y. Ma, J. Liang, S. Fu, X. Wan and Y. Chen, Small, 2009, 5, 1682. 